\title{
Prediction of local extinctions in piloted jet flames with inhomogeneous inlets using unstrained flamelets
}

\author{
Zhi X. Chen ${ }^{\mathrm{b}, 1}$, Ivan Langella ${ }^{\mathrm{a}, 1}$, Robert S. Barlow ${ }^{\mathrm{c}, *}$, Nedunchezhian \\ Swaminathan ${ }^{\mathrm{b}}$ \\ ${ }^{a}$ Aeronautical and Automotive Engineering, Loughborough University, Loughborough LE11 $3 T U$ \\ ${ }^{b}$ Department of Engineering, University of Cambridge, Cambridge CB2 1PZ, UK \\ ${ }^{c}$ Combustion Research Facility, Sandia National Laboratories, Livermore, CA 94550, USA
}

\begin{abstract}
Multi-regime turbulent combustion modelling remains challenging and is explored with occurrence of local extinction in this study. A partially premixed model based on unstrained premixed flamelets is used in this work to investigate a piloted jet flame configuration with inhomogeneous inlets. Three different cases are simulated, which differ in the bulk mean velocity that amounts respectively to about $50 \%, 70 \%$ and $90 \%$ of the blow off velocity measured experimentally. As the jet velocity approaches the blow off limit, local extinctions start to occur along the flame surface and thus these flames are challenging from a modelling prospective. Two different numerical approaches, involving scaled and unscaled progress variable respectively, are compared to elucidate their abilities and limitations to predict local extinctions and to deal with the three-stream problem at the pilot/coflow interface. The key modelling details for such predictions are indicated and discussed. LES results are systematically compared to two sets of
\end{abstract}

\footnotetext{
${ }^{*}$ Corresponding author

Email address: robertbarlow.bcr@gmail.com (Robert S. Barlow)

${ }^{1}$ These authors contributed equally to this work.
} 
experimental measurements available in the literature for the three flames. The differences observed in the two experimental datasets are also discussed with the help of LES results. Although both approaches show promising agreement for the flame statistics, the scaled progress variable approach better predicts the local extinctions. The unscaled approach shows to naturally handle the three-stream problem without additional treatment for the pilot/coflow interface, which is required for the scaled approach. Furthermore, computed scalar dissipation rate of mixture fraction is compared with the measurements showing good agreement for the conditions investigated. This further suggests that local extinctions can be predicted using unstrained flamelets if the correct scalar mixing and its dissipation are captured.

Keywords: Local extinction, Partially premixed flames, Inhomogeneous inlets, Unstrained flamelets, LES

\section{Introduction}

In modern engines the combustion is unduly turbulent due to the high flow velocities and the requirement of fast mixing to achieve high combustion efficiency. This mixing is imperfect in most combustion systems, so reactants are not homogeneously burned, like in modern gas turbine engines, where the lean premixed combustion is only partly achieved due to short resident times and to avoid potential flashbacks or contact with the surfaces $[1,2]$; or in diesel engines, where the rich premixed combustion is surrounded by diffusion flames [3]. These inhomogeneities cause local fluctuations that, in some conditions, lead to undesired local extinctions. The correct prediction and modelling of local quenching phenomena in inhomogeneous mixtures is thus of paramount importance for the design of 
stably-operating combustion devices.

Many experimental works have contributed to the understanding of local extinction phenomena. In turbulent non-premixed combustion as the mixing dominates the flame behaviour [4] the scalar dissipation rate (SDR) of mixture fraction has been identified as a key parameter for local extinctions [5]. When the SDR is below a lower limit, the mixing is not fast enough for the mixture to be ignitable. On contrary, if the SDR is above an upper limit and for a sufficiently long time, the diffusion losses can exceed the local heat release so that reactants can leak through the flame front causing a local extinction [6]. These views are supported by a number of experimental, see for example [7-9] and numerical, see for example [6, 10-12]. Also, the re-ignition was observed to occur on the lean side which is more reactive $[13,14]$. When the combustion is premixed the relation between scalar dissipation rate and extinction is more complex. The flame propagation induces strong flame/flow interaction, and mixing and turbulence can in turn affect the propagation speed. Depending on the level of this interaction the premixed combustion is divided into the well-known regimes $[15,16]$. Different mechanisms for local extinctions have been proposed in the past under premixed conditions. In initial works these extinctions were studied in terms of an imbalance between chemical, ignition and flow time scales [17-19]. Owing to the advance of diagnostic techniques, more recent experimental works (see for example [20-25]) show that local extinctions occur when the flame front moves into high vorticity regions in the shear layer, where the high strain rates break the flame front allowing fresh reactant to penetrate. Numerical works (see for example [2628]) have also shed light in this direction. Whether this situation leads to further local extinctions or reignition of the surrounding mixture, however, is not fully 
understood.

Large eddy simulation (LES) is becoming increasingly popular because of its ability to capture local transient phenomena. In LES the large scales are resolved with sub-grid scale (SGS) models to mimic the effect of unresolved processes [29]. As combustion is essentially a small-scale phenomenon, i.e., the flame thickness is usually smaller than the LES grid size, it requires sub-grid modelling. Many combustion models are now available for LES with their own advantages and limitations (see $[30,31]$ for a review), and among these model flamelet-based ones are arguably still the most popular owing to their efficiency, robustness and reasonably good accuracy. While it is difficult to conduct a thorough review on the many variants of the flamelet model (early contributions include [32-34], etc.), most of these models have been validated using flames under stably burning conditions. Exceptions exist for the Sandia flames F and E [13] where moderate and severe local extinctions were observed, and Ihme et al. [12] successfully modelled these flames using a flamelet/progress variable (FPV) approach [35]. It was clearly demonstrated in [12] that the steady flamelet formulation was not able to capture the local extinctions and including the unsteady effects of the mixture fraction scalar dissipation rate (SDR) was essential in the diffusion flamelet calculations. A simple interpretation recognises that the effects of flow on the reactive fields are not considered in a single steady diffusion flamelet calculation for a fixed representative strain rate. Consequently, for a given flammable mixture fraction the steady flamelet model would give the same thermochemical state regardless of the local flow conditions, which could both sustain and quench a flame depending on the mixture fraction SDR. When it comes to premixed flamelets, however, the argument becomes quite different. Although it has been demonstrated by many 
groups (e.g. [36-39], etc.) that the conventional diffusion jet flames can also be modelled using a collection of premixed flamelets with varying equivalence ratios, whether the SGS straining effects should be included remains as an open question. The classical view for which stretch is most effective at scales smaller than the Gibson scale [40] has been contradicted by a number of recent works [4144] showing that small eddies do not have sufficient energy or lifespan to impart significant changes to the flame structure. At the resolved scales, it is arguable that the effects of flow on the flame, at least to some extent, are taken into account through the transport equation for progress variable. However, this remains to be shown in a LES test case where there is strong local extinction, which is the objective for this study.

In real-life applications the combustion mode is often mixed or partially premixed [45], and the mechanisms leading to local extinctions are even more complex and seldom studied. Recent experimental studies by Meares et al. [46] and Barlow et al. [47, 48] tackled these issues by performing advanced diagnostics into a piloted jet flame with compositionally inhomogeneous inlets. The Rayleigh/Raman/LIF measurements not only revealed the rich physics of local extinctions under various conditions but also provided an extensive dataset for model validations. The high-spatial-resolution measuring techniques used made additional quantities available such as progress variable, joint scalar statistics as well as dissipation rates, most of which was first of its kind. In these experiments the occurrences of local extinctions along the flame surface were observed to consistently increase with the bulk velocity. Thus, with the rich data available these experiments present a good case for assessing the capabilities of current turbulent combustion models for predicting local extinctions in multi-regime 
flames, and to motivate further development of these models. Previous numerical attempts include transported probability density function (PDF) with stochastic fields [49], Lagrangian particles [50] and multi-environment [51] approaches, diffusion flamelet based models [52-54], multiple mapping conditioning [55] and hybrid approaches $[56,57]$. The performance of premixed flamelet based models remains unclear and none of these studies systematically focused on the prediction of location extinctions.

To this end, a recently formulated model $[58,59]$ based on unstrained premixed flamelets is used in this work to simulate three Sydney/Sandia piloted jet flames with inhomogeneous inlets [46, 47], namely the Lr75-57, Lr75-80 and Lr75-103 cases. The bulk velocities correspond to 50\%, 70\% and $90 \%$ of the blow-off velocity respectively, allowing one to examine the model performance with gradually increasing level of local extinctions. The combustion model used here has been validated in a number of different regimes and configurations for premixed $[43,60,61]$ and partially-premixed $[39,58,59,62]$ combustion, which include relatively high Karlovitz number flames. This study is the first attempt for this model to explore a case with local extinctions. For premixed flamelets based approaches, another issue which remains to be understood is the normalisation of the progress variable. Both scaled (values varying from 0 to 1 ) and unscaled formulations have been successfully used in the literature for various configurations, despite their respective underlying assumptions. Therefore, another objective here is to shed light on this by directly comparing the LES results computed using these two formulations. It is of particular interest to identify their advantages/disadvantages in a three-stream configuration with local extinctions like the cases investigated in the present study. 
This paper is organised as follows. The next section describes briefly the experimental burner along with its experimental setup. The LES model, subgrid closures, numerical method and grid used, and boundary conditions employed are described in section 3. The results are discussed subsequently followed by a discussion. Conclusions are summarised in the final section.

\section{Experimental case}

A sketch of the experimental setup for the Sydney/Sandia piloted burner with inhomogeneous inlets $[46,47]$ is shown in Fig. 1. The configuration consisted
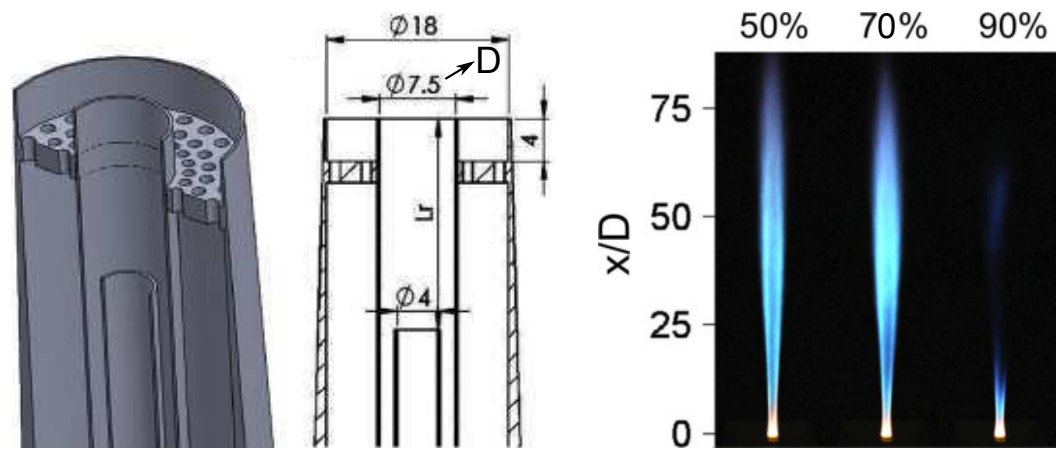

Fig. 1: Schematic of the burner [46] and typical averaged photographical images of the flames approaching blow-off (percentage indicates the $U_{b} / U_{\text {b.o. }}$ ratio).

Table 1: Experimental configurations selected for the numerical study.

\begin{tabular}{lcccccc}
\hline Case & $\operatorname{Lr}[\mathrm{mm}]$ & $U_{b}[\mathrm{~m} / \mathrm{s}]$ & $U_{f}[\mathrm{~m} / \mathrm{s}]$ & $U_{a}[\mathrm{~m} / \mathrm{s}]$ & $U_{p}[\mathrm{~m} / \mathrm{s}]$ & $U_{b} / U_{\text {b.o. }}$ \\
\hline Lr75-57 & 75 & 57 & 67 & 59.5 & 25.6 & $50 \%$ \\
Lr75-80 & 75 & 80 & 93.8 & 83.4 & 25.6 & $70 \%$ \\
Lr75-103 & 75 & 103 & 120.6 & 107.2 & 25.6 & $90 \%$ \\
\hline
\end{tabular}


of a polited methane/air jet issuing into a quiescent environment at room temperature of $298 \mathrm{~K}$ through a circular nozzle with a diameter of $D=D_{e}=7.5 \mathrm{~mm}$. Pure methane was injected into coflowing air through a concentric internal nozzle with a diameter of $D_{i}=4 \mathrm{~mm}$, which is located upstream of the main jet exit at a distance of Lr. The main jet nozzle is surrounded by a pilot with a diameter of $D_{p}=18 \mathrm{~mm}$ and a 5-gas reactant mixture $\left(\mathrm{C}_{2} \mathrm{H}_{2}, \mathrm{H}_{2}, \mathrm{CO}_{2}, \mathrm{~N}_{2}\right.$ and air $)$ was chosen to match the burnt gas temperature and composition of stoichiometric $\mathrm{CH}_{4} /$ air. The thickness of the rims of the two concentric nozzles is of $0.25 \mathrm{~mm}$ and the one for the external wall of the pilot is $0.2 \mathrm{~mm}$. The burner is placed at the centre of a squared wind tunnel of $1.5 \times 1.5 \mathrm{~m}^{2}$, where air flows at $15 \mathrm{~m} / \mathrm{s}$. Among the relatively large set of experimental configurations [47], only three will be considered in this work, for which the distance between the fuel nozzle exit and the burner entrance is $\mathrm{Lr}=75 \mathrm{~mm}$. These cases are summarized in Table 1 .

The experimental system yields an incomplete mixing between fuel and air at the burner entrance and thus these cases are interesting to explore effects of local inhomogeneities and for model validation. Moreover, the bulk velocity in these cases increases from $U_{b}=57,80$ and $103 \mathrm{~m} / \mathrm{s}$, where the bulk velocity is computed at the burner entrance using the mass flow rates of the fuel and air streams in the channel. These velocities correspond respectively to $50 \%, 70 \%$ and $90 \%$ of the blow off velocity, $U_{\text {b.o. }}$, observed experimentally. Their averaged photographical images are shown in the right side of Fig. 1. Note these images were taken for $\mathrm{Lr}=100 \mathrm{~mm}$ but the qualitative behaviour is similar. It is seen that the occurrences of local extinctions along the flame surfaces increase consistently from the low to the high $U_{b}$ configuration. Thus, these three cases are very useful for both model validation and to further explore the LES ability to predict local 
extinction phenomena in partially premixed combustion. The velocities of fuel, air and pilot streams are also reported in table 1. In particular, the pilot in these three configurations is placed $4 \mathrm{~mm}$ ahead of the nozzle exit. As will be discussed in more details in section 4, the pilot stream is not fully burnt which can be verified by the high levels of $\mathrm{CO}$ present in the experiments near the pilot exit. Moreover, the presence of a pilot makes the explored configuration a three-stream problem, which poses challenges for the numerical modelling and this is discussed in the next section.

The experimental measurements $[47,48]$ for the three cases include radial profiles of temperature, mixture fraction, velocity, major and minor species, and all corresponding rms values for different axial locations spanning from $x / D=1$ to $x / D=30$, where $x$ is the axial coordinate. More recently [48], 3D measurements of the SDR of the Bilger's mixture fraction became available, along with measurements of an oxygen-based progress variable and various correlations between SDR and progress variable. This new set of measurements is relevant for the analysis to be conducted in this study, as the SDR reveals further information about the local flame structure and local extinctions. The three flames of Table 1 were observed in the experiments to be very long, with parcels of fuel still burning at about $1 \mathrm{~m}$ distance from the nozzle exit. Moreover, it was observed that the combustion mode is mainly premixed in the near field region of the pilot stream then transitions to a diffusion mode downstream. This poses additional challenges for the numerical modelling, but at the same time is an opportunity to gain further understanding of the physics behind quenching. Note that flames Lr75-57 and Lr75-80 have been already simulated in a number of works discussed in the Introduction, while for the Lr75-103 case, which is the closer to blow-off condi- 
tions, the numerical attempts are rare [50]. Moreover, to the authors' knowledge this is the first study where SDR and progress variable measurements provided in $[48,63]$ are used to support a numerical analysis of this piloted jet burner with inhomogeneous inlet.

\section{LES methodology}

In the experiments [47], mixed mode combustion was observed showing premixed burning mode in the near-field and non-premixed mode in the downstream. Thus, a combustion model considering different mode contributions is required for the simulation of these flames. In this work, a tabulated chemistry approach based on unstrained premixed flamelets with non-premixed mode contribution to the subgrid reaction source term is used. This model has been validated for a wide range of conditions in various burner configurations and the formulation is only described briefly in this section as the elaborate details can be found in, e.g., [58] and [59]. The standard Favre-filtered LES equations for mass, momentum and total specific enthalpy (formation + sensible) conservation under the low-Mach number assumption are solved. The sub-grid scale (SGS) stresses are modelled using a transport equation for the subgrid kinetic energy $\widetilde{k}$ following previous works $[58,64]$. For combustion modelling, four additional scalar transport equations are solved and these equations along with their closure models are described next.

\subsection{Combustion modelling}

The reaction progress and turbulent mixing are tracked using respectively a progress variable, $c$, and a mixture fraction, $\xi$. The mixture fraction is defined using Bilger's formula [65]. The filtered mixture fraction and SGS variance, $\widetilde{\xi}$ and 
$\sigma_{\xi, \text { sgs }}^{2} \equiv \widetilde{\xi}^{2}-\widetilde{\xi}^{2}$, are solved using their transports equations, where the unclosed SGS scalar dissipation rate term, $\widetilde{\chi}_{\xi, \text { sgs }}$, is modelled using the linear relaxation model [30]. By contrast, the modelling of progress variable requires more care and depends on its definition, which is much less unified compared to that for mixture fraction. There are many ways to define the progress variable, e.g., using temperature, mass fraction of a single species, or a linear combination of few species, etc. The principle for these definitions is that the flamelet thermochemical states mapped into the lookup table must be monotonic in the progress variable space, and based on this requirement one could find an optimal definition as proposed in, for example, [66] and [67] using different optimisation approaches. While this choice of components in the definition has limited effects on the turbulent flame results (mainly lookup table interpolation errors), the considerably more influential or maybe controversial factor is the normalisation of progress variable. This aspect is seldom discussed, especially for partially premixed combustion, although both scaled (to unity) and unscaled progress variables are widely used in the literature. Bray et al. [68] pointed out the potential advantages and disadvantages of the normalisation via intuitively examining the unclosed terms in the progress variable transport equation derived from first principles. However, these arguments are still yet to be validated through direct a posteriori comparison for simulations using scaled and unscaled progress variables. Also, for the experimental perspective it is preferable to define a Raman species-based normalised progress variable [63], so that it varies from 0 to 1 between the reactants and products regardless of the local mixture fraction. To compare with the measurements, however, one does necessarily need to transport the same progress variable during the simulation because it can be reconstructed through post-processing. In 
this study, both scaled and unscaled progress variables, denoted as S-PV and UPV hereafter, are explored in the LES to shed some light on the potential effects discussed above. This leads to different transport equations and closure formulations, which are described in the following.

Following previous studies $[58,59,69]$, the sum of $\mathrm{CO}$ and $\mathrm{CO} 2$ mass fractions, $\psi \equiv\left(Y_{\mathrm{CO} 2}+Y_{\mathrm{CO}}\right)$, is used to define the progress variable. Depending on whether scaled or not, in laminar unstrained premixed flames with varying mixture fraction this definition reads

$c(\xi)=\frac{\psi}{\psi_{b}(\xi)}$

or $c=\psi$,

where the superscript ${ }^{b}$ denotes the burnt side value. Note that the U-PV is often denoted using $Y_{c}$ (see $[36,38]$ for example) and here $c$ is used for conciseness. Because both expressions show dependence on the mixture fraction, differentiating them in time and space results in additional terms while deriving their exact transport equations (see details in $[68,70]$ ). After careful mathematical manipulation and using appropriate approximations, the filtered progress variable and its SGS variance equations, in a common form for the S-PV and U-PV, are written as

$\bar{\rho} \frac{D \widetilde{c}}{D t}=\nabla \cdot\left[\left(\overline{\rho \mathcal{D}}+\frac{\mu_{t}}{\operatorname{Sc}_{t}}\right) \nabla \widetilde{c}\right]+\overline{\dot{\omega}^{*}} \quad$ and

$$
\begin{aligned}
\bar{\rho} \frac{D \sigma_{c, \mathrm{sgs}}^{2}}{D t}= & \nabla \cdot\left[\left(\overline{\rho \mathcal{D}}+\frac{\mu_{t}}{\mathrm{Sc}_{t}}\right) \nabla \sigma_{c, \mathrm{sgs}}^{2}\right]-2 \bar{\rho} \widetilde{\chi}_{c, \mathrm{sgs}} \\
& +2 \frac{\mu_{t}}{\mathrm{Sc}_{t}}(\nabla c \cdot \nabla c)+2\left(\overline{c \dot{\omega}^{*}}-\widetilde{c} \overline{\dot{\omega}^{*}}\right),
\end{aligned}
$$


where $\frac{D}{D t}$ denotes the substantial derivative, $\widetilde{\mathcal{D}} \approx \widetilde{\mu} / 0.7$ is the filtered molecular diffusivity which is assumed to be the same for all scalars, $\mathrm{Sc}_{t} \approx 0.7$ is the SGS Schmidt number and $\tilde{\mu}$ is the filtered dynamic viscosity computed via Sutherland's law. The SGS viscosity is closed as $\mu_{t}=C_{k} \bar{\rho} \Delta \sqrt{k}$, where $\bar{\rho}$ is the filtered density, $\Delta$ is the LES filter size approximated as the cubic root of the local cell volume, $k$ is the SGS kinetic energy and $C_{k} \approx 0.1$ [29].

The reaction rate term in Eq. (2) before filtering, $\dot{\omega}^{*}$, includes the additional derivative terms discussed earlier, and by neglecting the cross dissipation term [ 68 , $71]$ it can be written as

$\dot{\omega}^{*} \approx \dot{\omega}_{\mathrm{fp}}+\dot{\omega}_{\mathrm{np}}$

with the terms on the RHS given by

$\dot{\omega}_{\mathrm{fp}}=\dot{\omega}_{\psi} / \psi_{b} \quad$ and $\quad \dot{\omega}_{\mathrm{np}}=\rho \chi_{\xi} \frac{c}{\psi_{b}} \frac{d^{2} \psi_{b}}{d \xi^{2}} \quad$ for the S-PV;

$\dot{\omega}_{\mathrm{fp}}=\dot{\omega}_{\psi} \quad$ and $\quad \dot{\omega}_{\mathrm{np}}=0 \quad$ for the U-PV,

where $\overline{\dot{\omega}}_{\psi}$ is the reaction rate of $\psi$ and $\chi_{\xi} \equiv \mathcal{D}|\nabla \xi|^{2}$ is the mixture fraction SDR. In terms of physical interpretation, $\overline{\dot{\omega}}_{\text {fp }}$ represents the contribution of stratified premixed combustion (with low mixture fraction SDR), and $\overline{\dot{\omega}}_{\text {np }}$ takes the form of the diffusion flamelet expression [32] signifying the non-premixed mode contribution.

The modelling of the filtered premixed term is similar for both approaches:

$\overline{\dot{\omega}}_{\mathrm{fp}}=\bar{\rho} \int_{0}^{1} \int_{0}^{1} \frac{\dot{\omega}_{\mathrm{fp}}(\zeta, \eta)}{\rho(\zeta, \eta)} P(\zeta, \eta) \mathrm{d} \zeta \mathrm{d} \eta$,

where $\zeta$ and $\eta$ are sample space variables for $c$ and $\xi$ respectively, and $P(\zeta, \eta)$ is their SGS joint probability density function (PDF). This PDF represents the 
subgrid statistics of $\xi$ and $c$, and is commonly approximated as a joint distribution of two independent Beta functions, $P_{\beta}(\zeta)$ and $P_{\beta}(\xi)$. This is followed in this study although the assumption of statistical independence is to be questioned, as suggested by recent experimental findings [48, 63], which will be explored in a future study. While other presumed PDFs are possible, beta functions were shown to be preferable for LES of extinction and re-ignition problems, where unsteady effects are important $[6,72]$. It is worth noting here that Beta distribution strictly requires the variable to vary from 0 to 1 and thus for the U-PV approach both the filtered value and variance have to be scaled before accessing the flamelet lookup table. The Beta PDF for the progress variable is then calculated as $P_{\beta}(\zeta)=P_{\beta}\left(\widetilde{c} / \psi_{b}, \sigma_{c, \mathrm{sgs}}^{2} / \psi_{b}^{2}\right)$, where $\psi_{b}$ is often obtained as $\psi_{b}(\widetilde{\xi})$ implying that the effect of SGS mixture fraction variation on $\psi_{b}$ is neglected. Also, such a scaling is somewhat $a d$ hoc and can be problematic numerically for mixture fraction near the flammability limits where both $\psi$ and $\psi_{b} \rightarrow 0$. For the S-PV, however, this issue does not exist since the scaling is done prior to the LES. On the other hand, the scaled approach can have its own issue when there is a third stream involved such as the configuration of interest here, and this is explained in more detail later in Section 3.4. We shall see these influences due to the scaling of progress variable in more detail later while discussing the results in Section 4.

For the non-premixed term in Eq. (4), past studies using the S-PV have shown that $\overline{\dot{\omega}}_{\text {np }}$ can be of the same order of $\overline{\dot{\omega}}_{\text {fp }}$, resulting in a significant impact on the overall reaction rate at the base of lifted jet flames $[73,74]$. Thus, this term should not be neglected generally when the S-PV is used, at least for cases where there is strong scalar dissipation near the stoichiometry. Following previous studies [58, 
59], here the non-premixed term is modelled as

$\overline{\dot{\omega}}_{\mathrm{np}} \approx \bar{\rho} \widetilde{c} \widetilde{\chi}_{\xi} \int_{0}^{1} \frac{1}{\psi^{b}(\eta)} \frac{\mathrm{d}^{2} \psi^{b}(\eta)}{\mathrm{d} \eta^{2}} P(\eta) \mathrm{d} \eta$,

where $\widetilde{\chi}_{\xi}=\widetilde{\mathcal{D}}(\nabla \widetilde{\xi} \cdot \nabla \widetilde{\xi})+\widetilde{\chi}_{\xi \text {,sgs }}$ is computed as sum of resolved and SGS contributions. For the U-PV, this term does not appear and the progress variable is transported like a species mass fraction. It is worth noting here that in neither approaches the non-premixed flame structure is computed directly from the thermodynamic state of the flamelets. Instead, the large-scale mixing effects are considered through the mixture fraction (equivalence ratio for premixed flamelets) and its subgrid PDF for the SGS level variations. Since each premixed flamelet is computed independently, it may be inadequate to represent the detailed structure in the mixture fraction space in the limit of classical non-premixed flames. Similarly, it is difficult to use non-premixed flamelets for purely premixed combustion. In the partially premixed context, Vreman et al. [75] found that premixed flamelets provide certain advantages in a test case similar to the present configuration. Further discussion on this topic is beyond the scope here and details can be found in [75].

The reaction related term, $\overline{c \dot{\omega}^{*}}$, in Eq. (3) is computed in a manner consistent with Eq. (5). The subgrid SDR of progress variable, $\widetilde{\chi}_{c, \mathrm{sgs}}$, is modelled differently than the that of the mixture fraction because the combustion effects must be included [76]. A well validated algebraic expression [39, 58, 60, 61, 77] with dynamic procedure for the model constant [78] is used for this study. It is worth noting that this model constant varies significantly with axial position in the three flames under investigation, which is probably due to the nature of these flames transitioning from premixed to diffusion mode [47]. A fixed model constant was observed to give incorrect prediction of temperature and other quantities either up- 
stream or downstream, suggesting that the SDR of progress variable varies along the flame in nature. More details on this point will be given in Section 4.

The temperature is obtained using $\widetilde{T}=T_{0}+\left(\widetilde{h}-\widetilde{\Delta h}_{f \text {,mix }}^{0}\right) / \widetilde{C}_{p \text {,eff }}$, where $T_{0}=$ $298.5 \mathrm{~K}$ is the reference temperature and $\widetilde{h}$ is transported specific total enthalpy. The filtered formation enthalpy and effective heat capacity [73] at constant pressure of the gas mixture, $\widetilde{\Delta h}_{f \text {,mix }}^{0}$ and $\widetilde{C}_{p \text {,eff }}$, are calculated in a similar manner to Eq. (5) and then tabulated in the lookup table. This avoids the need to tabulate all the species mass fractions, which can cause computer memory issue for large chemical mechanisms. The mixture density is computed using the state equation, $\bar{\rho}=\bar{p} \widetilde{W}_{\text {mix }} /\left(R_{0} \widetilde{T}\right)$, where $\bar{p}$ is the modified pressure [29], $\widetilde{W}_{\text {mix }}$ is the mixture molecular weight, which is also tabulated, and $R_{0}$ is the universal gas constant.

\subsection{Numerics}

The OpenFOAM libraries [79] are used to solve the low-Mach number reacting flow equations on unstructured grids using the finite volume method with second-order accuracy in space and time. The numerical domain, sketched in Fig. 2, extends to $100 \mathrm{~mm}$ upstream of the jet exit in order to simulate the inhomogeneous mixing between fuel and air. The fuel nozzle exit is located at $75 \mathrm{~mm}$ upstream corresponding to the Lr75 configurations in the experiments. In most LES studies on this burner so far (e.g., [53, 55, 56]), a separate mixing simulation was first performed for the upstream pipe (marked as mixing region in Fig. 2) and then the pipe outflow solution was used as the inlet boundary condition for the reacting flow simulation. Despite the complexity of handling the data consistently from one simulation to another, this approach considerably reduces the computational cost. However, such an I/O intense simulation is not favourable for larger cases in industrial practice. In this work, the mixing region is computed in the 
LES along with downstream reacting region as shown in Fig. 2. Both the fuel and air flows are fully developed before entering the mixing pipe and thus accurately prescribing their turbulence levels at the LES inlets is crucial, since the inhomogeneous mixing which dictates the burning mode and flame stability behaviour [46] is largely affected by the inlet turbulence of the two streams. To address this, a synthetic eddy turbulence generator [80] is used and the details are described in Appendix A. Note that only velocity fluctuations are imposed at the inlets as fuel and air enter the numerical domain separately. The mixture fraction fluctuations, i.e., compositional inhomogeneities, are generated by solving the LES equations.

The cylindrical region downstream of the nozzle exit spans $50 D$ and $15 D$ in the axial and radial directions respectively. The numerical grid consists of about

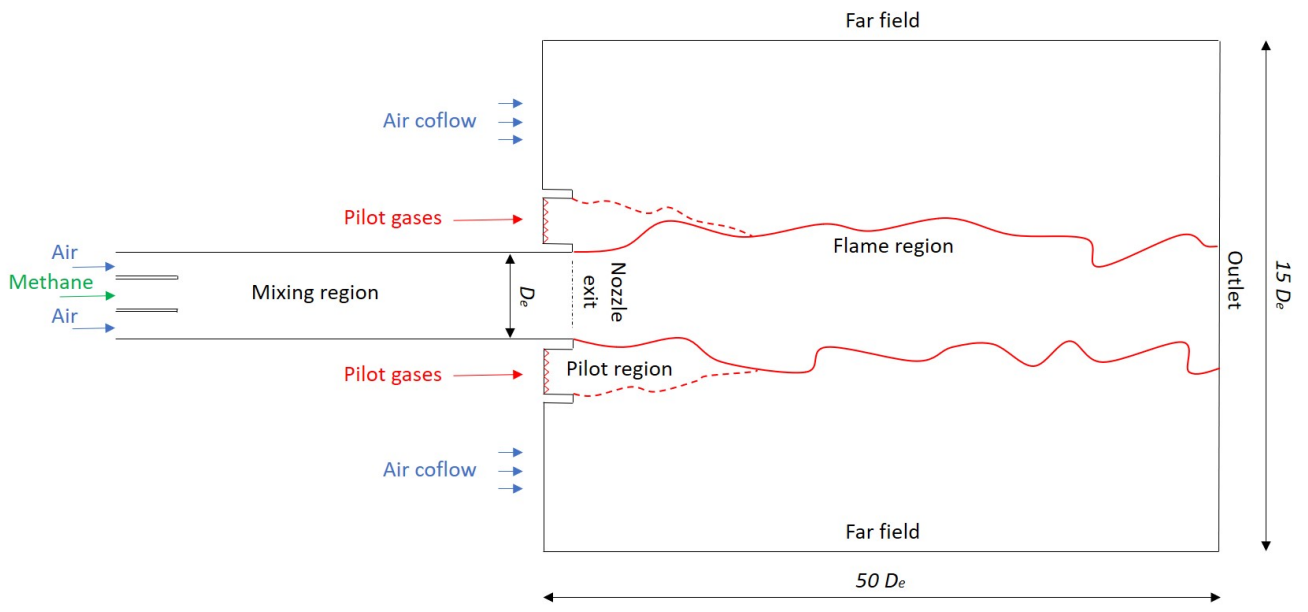

Fig. 2: A sketch of the computational domain (not in scale) used for the LES.

3.2M hexahedral cells with most of them clustered near the nozzle exit and in the shear layer. There are 88 cells spanning the nozzle diameter, non-uniformly 
spaced to accumulate close to the walls and at the interface between fuel and air inlets. A grid sensitivity analysis was performed using three additional grids respectively having 2.2, 3.2 and $8 \mathrm{M}$ elements, mainly differing in the region of the pipe where the mixing happens. Although all three test grids satisfy Pope's criterion for turbulent kinetic energy [29], the analysis showed that the coarser grid $(2.2 \mathrm{M})$ are unsatisfactory in predicting the correct level of mixing upstream, which was verified by comparing results for mixture fraction with the experimental data close to the nozzle exit. No significant difference was observed between the $8 \mathrm{M}$ and $3.2 \mathrm{M}$ grid results and thus the latter is used.

According to the experimental data, flat velocity profiles of 15 and $25.6 \mathrm{~m} / \mathrm{s}$ are given at the coflow and pilot inlets respectively. Mixture fraction is specified to be 0 for the air streams, 1 for the fuel stream and stoichiometric $(0.55)$ in the pilot stream. The progress variable is zero for all inlets except for the pilot, where it has the value of 1 for S-PV approach or $\psi_{b}\left(\xi_{s t}\right)$ for the U-PV approach. The measurements at $x / D=1$ indicate that the pilot mixture slightly deviates from the fully burnt condition in the experiments and the sensitivity of LES results to this pilot boundary condition is detailed in the Supplementary Material. Standard far field and pressure outlet conditions are used for the side and outlet boundaries, respectively. A two-layer wall function model [81] is employed for the wall boundaries. Each simulation took about 24 hours using 240 processors on a wall clock for 12 flow-through-time (estimated using 30D/ $U_{b}$ ), of which the last 6 were used to collect the statistics.

\subsection{Flamelet table}

The flamelet reaction rate, $\dot{\omega}(c, \xi)$, is obtained from unstrained planar laminar premixed flames with $\xi$ spanning the entire flammability limits for atmospheric 
methane-air combustion. These flamelets are computed using PREMIX [82] and GRI-Mech 3.0 mechanism. The formation enthalpy, effective heat capacity and molecular mass of the mixture are computed consistently with Eq. (5) and are also included in the lookup table. The filtered reaction rate is zero outside the flammability limits whereas the other quantities are linearly interpolated to the air and fuel values [74]. For the lookup table, uniformly spaced 100 and 50 points are used for $0 \leq \widetilde{c} \leq 1\left(0 \leq \widetilde{c} \leq \psi_{b}\right.$ for the U-PV approach) and $0 \leq \sigma_{c, \mathrm{sgs}}^{2} \leq \widetilde{c}(1-\widetilde{c})$ spaces respectively, and 200 points are used for $0 \leq \widetilde{\xi} \leq 1$ with refinement within the flammability limits. Following [58], 15 points concentrated near 0 are used for $0 \leq \sigma_{\xi, \mathrm{sgs}}^{2} \leq \widetilde{\xi}(1-\widetilde{\xi})$.

\subsection{Management of the three-stream problem}

At the interface between the pilot and the air coflow stream, reactions cannot occur because air is mixed with burnt mixture. However, the S-PV approach described earlier can still give reactions because the mixture is within the flammability limits and the progress variable is lower than 1 because it has been diluted by the coflowing air stream. In other words, the described model cannot distinguish whether the progress variable has intermediate value because of actual combustion or simple mixing between burned gases and air. One potential way to overcome this issue is to use an unscaled progress variable and the U-PV approach introduced in section 3.1. This is because the unscaled progress variable is more directly linked with the adiabatic temperature and mixing in partially premixed situations as described in [83]. At the pilot/coflow interface mixture fraction and progress variable are subject to the same amount of mixing (at least when Schmidt numbers are the same), and thus the unscaled progress variable is reduced (from the pilot to the coflow) proportionally to the reduction in mixture fraction so that 
the burnt state remains the same. This is not the case for a scaled progress variable where the decrease from the unitary value to a smaller value unavoidably implies a change of burning state. This peculiarity of the U-PV approach is tested in this work.

To avoid the spurious reactions in the air-coflow/pilot mixing layer, a fuel tracker is used for the S-PV approach. The filtered fuel mass fraction, $\widetilde{Y}_{\mathrm{CH}_{4}}$ is transported with its consumption rate tabulated in the lookup table. Zero value of $\widetilde{Y}_{\mathrm{CH}_{4}}$ is specified for the air inlet, pilot and coflow boundaries, and unity is given for the fuel inlet. Therefore, the non-reacting pilot/coflow mixing layer can be distinguished from the reacting pilot/jet mixing layer depending on the

presence of fuel: if $\widetilde{Y}_{\mathrm{CH}_{4}}>0$, the reaction rate source terms in Eqs. (2)-(3) and the thermochemical state are obtained from the lookup table; otherwise, the reaction rate is set to be 0 and the other properties are computed using a simple mixing rule: $\phi=\widetilde{c} \phi_{p}+(1-\widetilde{c}) \phi_{\text {air }}$, where $\phi_{p}$ and $\phi_{\text {air }}$ are the values of $\phi$ in the pilot and coflow streams respectively. It is worth noting that without the treatment described above involving the fuel tracker and mixing rule, the pilot stream was found to be unstable showing large Kelvin-Helmholtz vortices flapping along the coflow/pilot interface. Consequently, this resulted in completely incorrect predictions in the region $1<x / D<10$ where the pilot effects are critical.

\section{Results and discussion}

\subsection{General flame trends \& combustion modes}

In the experimental works [46-48] the flame was observed to propagate in a stratified, inhomogeneous mixture close to the pilot region. This behaviour was also seen in the numerical work [53] arguing that the inhomogeneous inlets place 
flammable, near-stoichiometric pockets close to the pilot. Moving downstream the flame was observed to transition into a diffusion mode. Moreover, effects of differential diffusion were hypothesised [48] in this region.

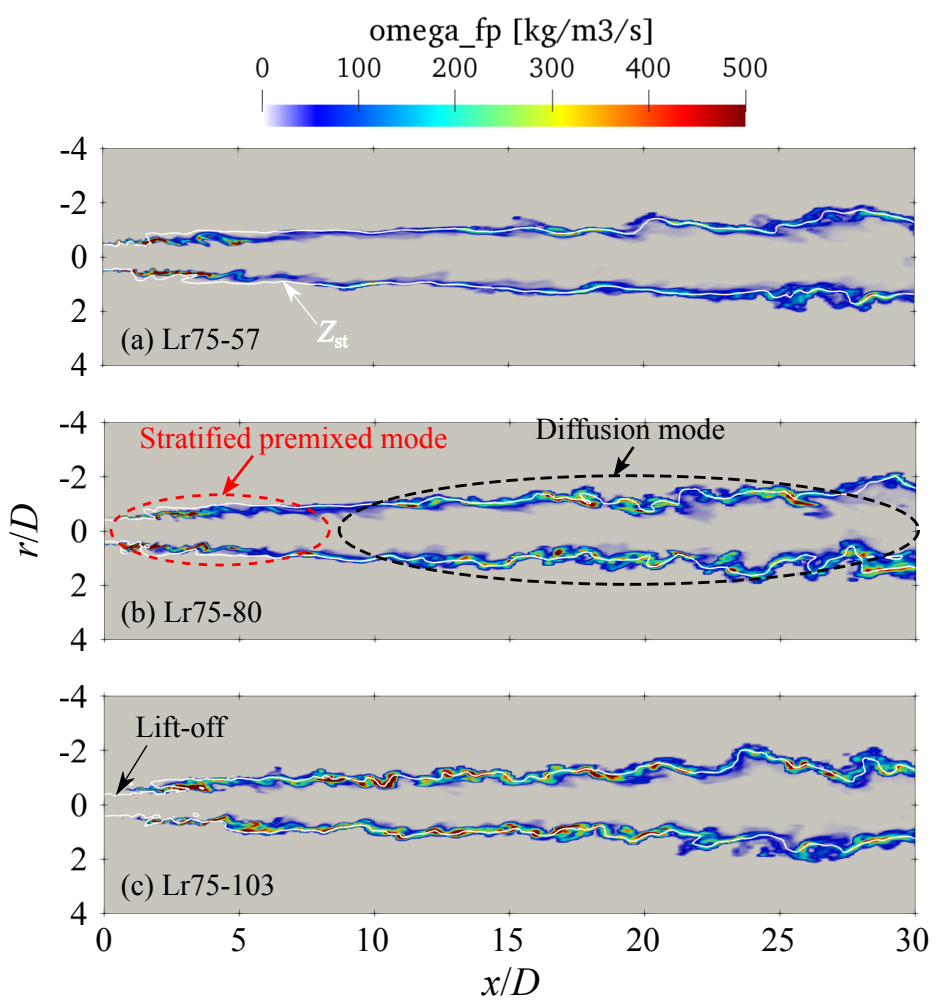

Fig. 3: Mid-plane contours of instantaneous premixed reaction rate contribution $\overline{\dot{\omega}}_{\text {fp }}$ for cases(a) Lr75-57, (b) Lr75-80 and (c) Lr75-103. White iso-line indicates stoichiometric mixture fraction.

The combustion model with S-PV approach used in this study distinguishes between premixed and non-premixed combustion modes of the reaction rate, see Eq. (4), and thus these two contributions from the LES can be compared. Instantaneous contours of premixed and non-premixed contributions to the reaction rate of progress variable for the three cases in Table 1 are shown in Figs. 3 and 4 respectively. It is worth recalling from Eq. (6) that the non-premixed contribution 


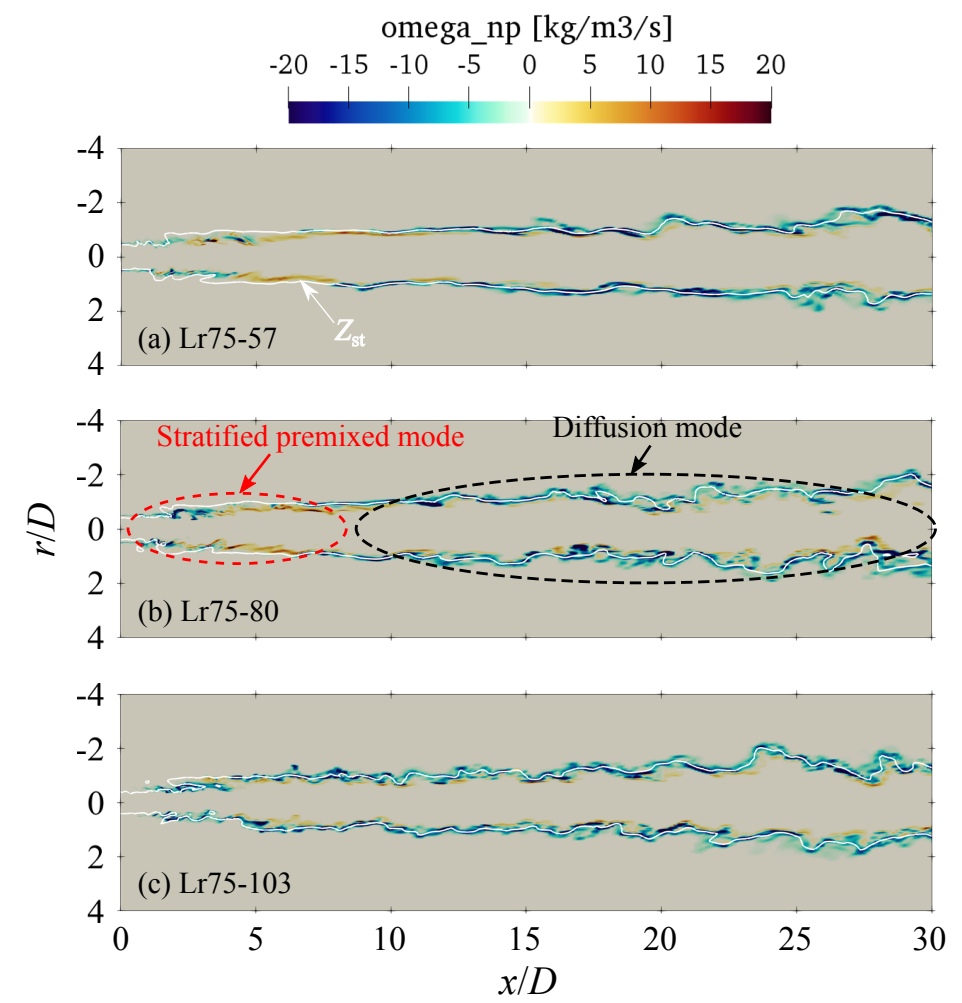

Fig. 4: Mid-plane contours of instantaneous non-premixed reaction rate contribution $\overline{\dot{\omega}}_{\text {np }}$ for cases(a) Lr75-57, (b) Lr75-80 and (c) Lr75-103. White iso-line indicates stoichiometric mixture fraction.

does not represent the reaction rate which one would have in a diffusion flame, but rather a correction term for the reaction rate to account for non-premixed combustion effects. Thus, this term can take both positive and negative values. For all three cases, the flame observed in Fig. 3 is very long with reactions occurring throughout the domain plotted. No spurious reactions are present at the pilot/coflow interface, suggesting that the three-stream problem is correctly handled in the modelling. A careful examination of Figs. 3 and 4 can further lead to the following observations: 
- the flame is lifted and the lift-off height increases with the bulk velocity as one would expect. This could not be observed in the experiments as it is masked by the pilot. At the flame base, within $2 D$ after the jet exit, the reaction appears mainly in the lean side of stoichiometry.

- the combustion mode transition observed in the experiments [47], from stratified premixed to diffusion mode within $10 D$ downstream of the jet exit, is also shown in the simulations for all three cases. As marked in Fig. $3 b$ using a red circle, the reaction layer in the upstream stratified premixed burning region does not necessarily follow the stoichiometric mixture fraction iso-line and most reactions occur in the rich side suggesting a rich premixed burning mode, which is consistent with the experimental observations (see Fig. 11). This behaviour is also seen for the other two flames in Figs. 3a and 3c. Moving towards downstream, after approximately $10 D$ the reaction zone falls closely within the vicinity of the stoichiometry as in a typical diffusion jet flame. As the jet bulk velocity increases, this transition seems to happen earlier, i.e., more upstream in the flame, which is probably due to the shorter pilot in the high velocity flames (see detailed discussion later for Fig. 5);

- in Fig. 4 the non-premixed contribution to the filtered reaction rate is observed to have a maximum which is an order of magnitude smaller than that of its premixed counterpart in Fig. 3. It is interesting to note that from the rich premixed to non-premixed mode the value of $\overline{\dot{\omega}}_{\text {np }}$ experiences a sign change from predominantly positive to negative. This implies that this term could be a potential combustion mode indicator in addition to the 
convectional Takeno flame index [84];

\subsection{Flame structure}

The two approaches described in section 3.2, S-PV and U-PV, are compared with experimental data for validation purposes and to further describe the structure of the flame. Only representative quantities are shown here and a comprehensive comparison for velocity, major and minor species is available in the Supplementary Material. While most past numerical studies had a smaller domain of interest (up to 15 or $20 D$ ), the entire measurement range is covered for a thorough comparison here using four locations: $x / D=1,5,15$ and 30 . It is worth noting that the pilot stream has a significant impact on the prediction of first and second order statistics of the flames under investigation. This is because the flame is lifted, as observed in Fig. 3, and thus the length of the pilot stream will determine the correct anchoring of the flame, consequently affecting the statistics. This is complicated by the fact that the interface between pilot and coflowing air exhibits some sort of flapping which could be a combination of Kelvin-Helmoltz and RayleighTaylor instabilities due to the differences in both velocity and density between the two streams. The length of the flapping behaviour seems to be dependent on the jet bulk velocity as shown in Fig. 5. Since the pilot and coflow velocities remain unchanged as the configuration changes from Lr75-57 to Lr75-103, the increase in the $U_{b}$ results in a shorter pilot and to some extent enhances the flapping. Thus, the correct prediction of this instability is important for correctly predicting the region near the pilot, given the lifted nature of the flame. This behaviour is only discussed here because of its relevance for the prediction of statistics, while its causes are still unclear and will be a subject worthy of further numerical and experimental investigations. 


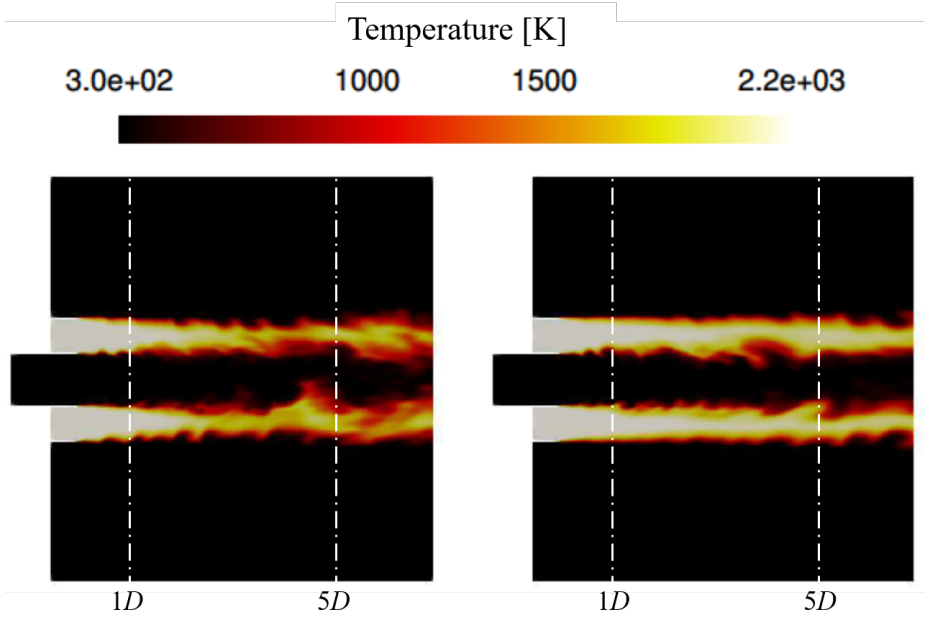

Fig. 5: Mid-plane contours of temperature in the region near the pilot for cases Lr75-103 (left) and Lr75-57 (right).

\subsubsection{Comparison of main statistics}

Figure 6 shows measured and computed results of Bilger's mixture fraction [65]. Density-weighted measurements both from [47] and [48] are shown to compare with the LES results from the U-PV and S-PV approaches. The correct prediction of this quantity is essential for the inhomogeneous cases investigated, as variation of mixture fraction substantially affect the temperature field. As shown in the figure both LES approaches predict mean and rms statistics with very good accuracy. The predictions from the two approaches are very similar because mixture fraction is only indirectly affected by the different combustion modelling through the temperature (or density) field. Here the computed penetration of the jet core (see centreline value at $r=0$ ) is in excellent agreement with the measurement. This is to be compared with the numerical studies (e.g., $[52,85])$ using separately computed solutions for the inhomogeneous mixing where the downstream 

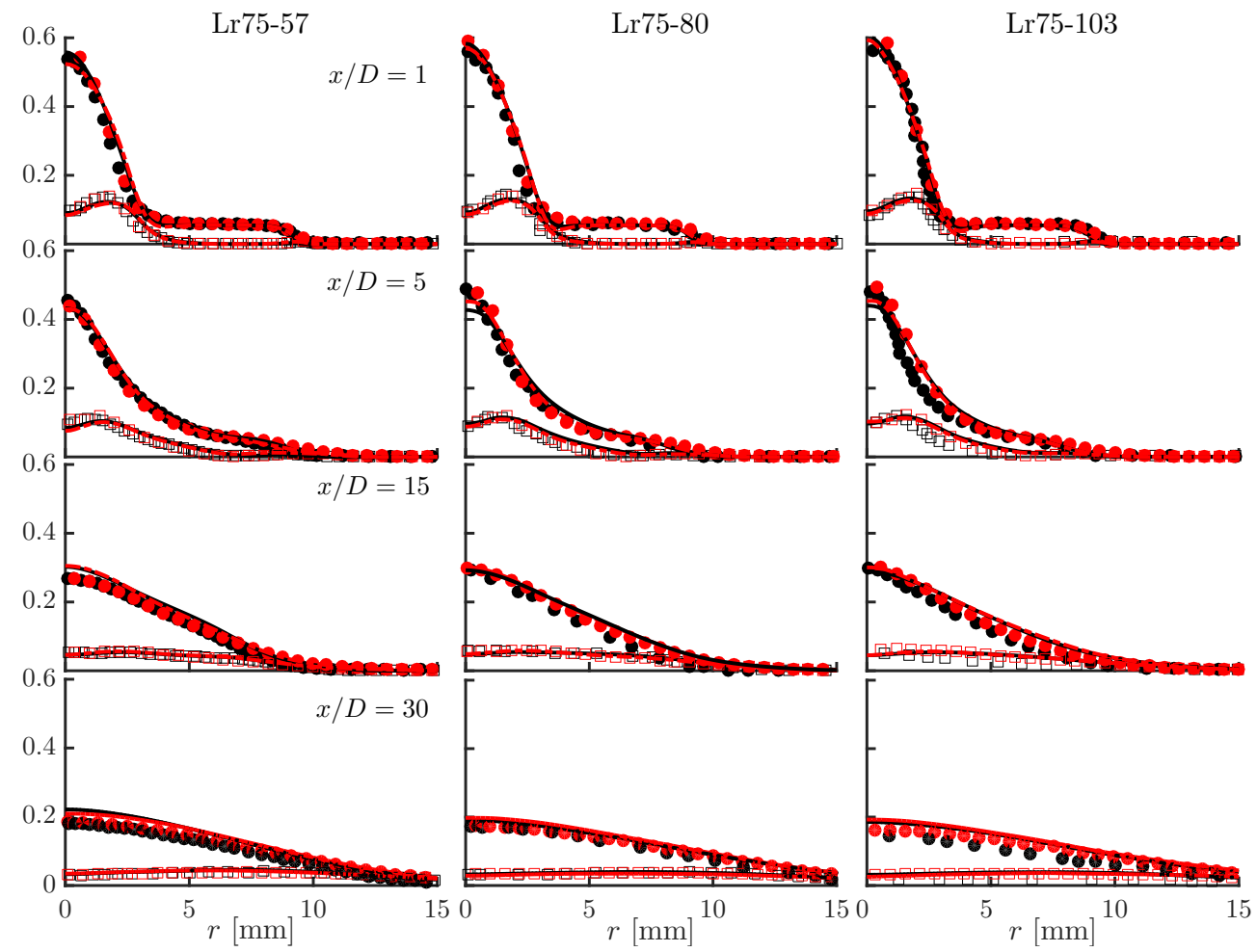

Fig. 6: Radial profiles of mixture fraction and its rms from experiment (mean: • [47], • [48]; rms: $\square$ [47], $\square$ [48]) are compared to LES results (S-PV, — and U-PV, - · -) at various axial locations.

jet penetration is considerably overestimated although the near-field mixing is accurately captured. This is because the hybrid RANS/LES approach used here for the upstream mixing (described in Appendix A) offers a physically more consistent modelling for the evolution of the compositional inhomogeneity in both the upstream pipe and downstream jet flows. Here the rms is also very well predicted as compared to the measurements in [48], which are generally higher than those measured earlier in [47] especially for large radial positions. This difference in the measurement data could be related to a slight difference between the two set of experiments in the vertical misalignment (a small vertical misalignment of the 

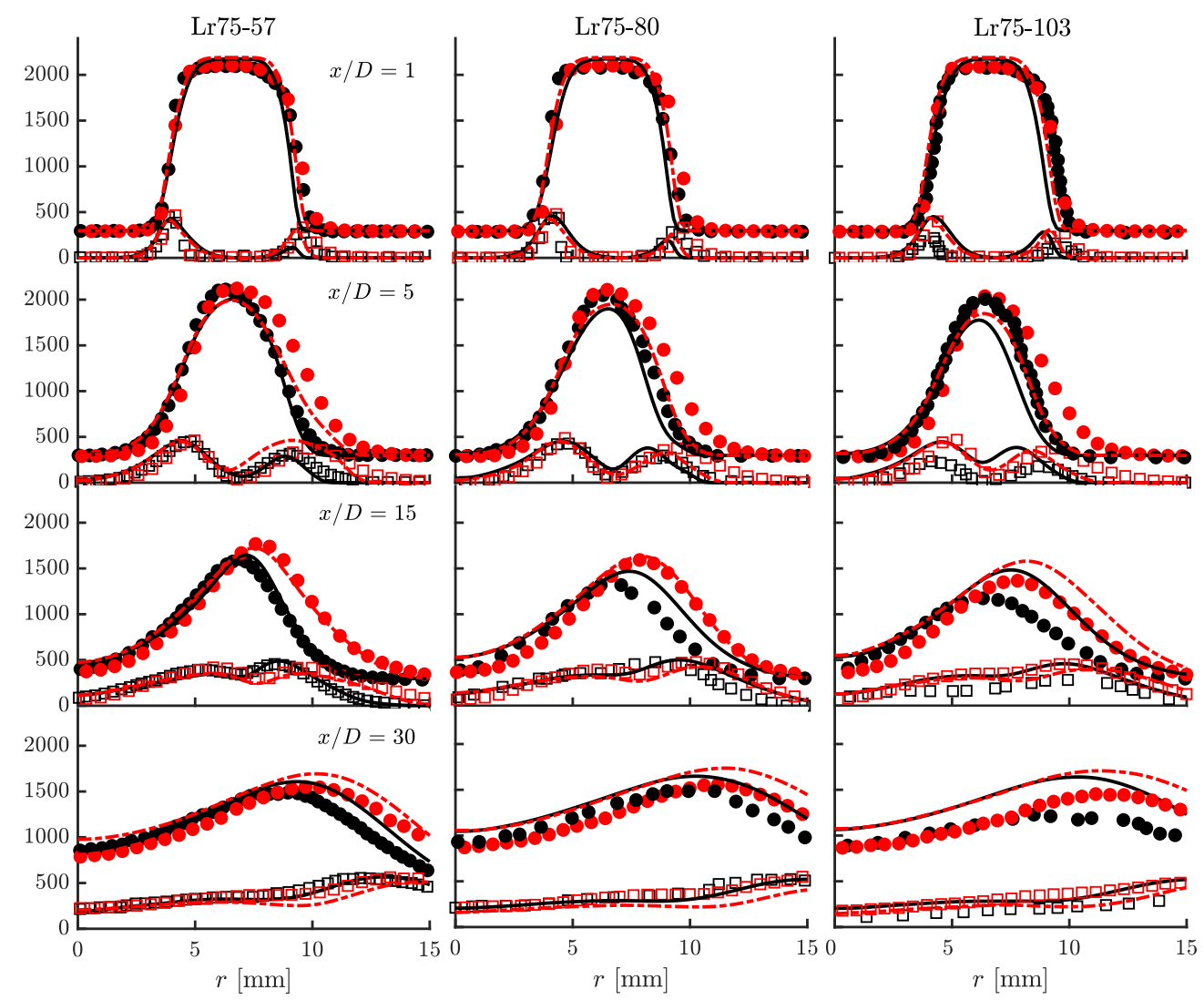

Fig. 7: Radial profiles of temperature and its rms from experiment (mean: • [47], • [48]; rms: $\square$ [47], $\square$ [48]) are compared to LES results (S-PV, — and U-PV, - · -) at various axial locations.

burner could cause asymmetry in boundary layer development along the exterior of the burner, which would alter shear layer development between coflow air and pilot products). Nevertheless, the mixing fields from the two sets of measurements $[47,48]$ are also very similar, and both show good agreement with the computational results. This lends the support for the examination of the combustion model performance, which is discussed next.

The mean and rms temperature profiles are compared with the experimental 
data in Fig. 7 in a similar manner to Fig. 6. Differently from the mixture fraction and except for the $x / D=1$ location, the temperature measurements in [48] differ significantly from those in [47] for radial positions $r>6 \mathrm{~mm}$. The overall trend is that the temperature distribution measured in [48] for the non-reacting coflow/pilot layer is shifted towards the outer regions at $x / D=5$ while the peak temperature and its radial position remains almost the same. This is likely to be related to the shear layer instability discussed earlier in Fig. 5, which was quite sensitive to the vertical alignment in the experiments resulting in a very different penetration of the pilot. Consequently, the downstream reaction was significantly affected and the stronger pilot penetration supports the flame further downstream in [48] leading to a higher peak mean temperature at $x / D=15$ for all three flames, compared with that measured in [47]. It should also be noted that less location extinctions were observed in [48] which could also contribute to the higher temperatures in the downstream. Interestingly, a very similar difference is found between the two sets of LES results using U-PV and S-PV approaches. Although both LES results show a good overall agreement with the measurements, the UPV model gives a outwardly shifted temperature distribution at the downstream locations, $x / D=5,15$ and 30. Similar to the experimental shift, the computed peak temperature also increases at the two location from the S-PV to U-PV. However, despite the coincidental match with experiments, this shift is unlikely to be a pilot effect due to geometrical arrangement since exactly the same numerical grid and boundary conditions are used for the two models. Instead, the change in the numerical results is related to the different normalisation procedures used for the U-PV and S-PV approaches.

Figure 8 compares the typical mid-plane contours of the normalised progress 
variable using the two models at arbitrarily chosen times for the Lr75-80 case. Here it is worth recalling that in the S-PV approach the normalised progress variable is transported using Eq. (2), whereas normalisation is performed after solving the unscaled equation using $\widetilde{c} / \psi_{b}(\widetilde{\xi})$ for the U-PV. When the mixture fraction approaches the flammability limits, $\psi_{b}(\widetilde{\xi})$ becomes very small, which gives rise to substantial numerical errors. This is particularly problematic on the lean side of the flame as can be seen in Fig. 8a, where large values are present outside the lean flammability limit. By contrast, the S-PV approach gives a physically consistent distribution of the normalised progress variable with the correct boundedness. Indeed, one may argue that since the normalised progress variable in the U-PV approach is only used for looking up the table, the variation of the tabulated quantities is small near the flammability limits and thus an overestimated progress variable in these regions has a limited effect on the LES solution. This is only true for the reaction rate source term while significant deviation can occur for the thermochemical quantities (filtered formation enthalpy and effective heat capacity) and thus the temperature. When the flame is burning with a strong support from the pilot, for example at $x / D=1$ and 5, this effect is negligible and the two models give very similar results for all three cases. However, as the pilot is diluted (the burnt gas becomes leaner) by the air coflow in the downstream, the normalised progress variable for table lookup is over-estimated in the U-PV approach (see Fig. 8a), which enhances the downstream burning. This explains the higher lean-side mean temperature given by the U-PV for the downstream locations $x / D=15$ and 30 observed earlier in Fig. 7. Furthermore, evident local extinction holes are seen in Fig. 8b for the S-PV between $x / D=15$ and 20 as in the experiments $[46,47]$ but none can be observed for the U-PV in Fig. 8a. 


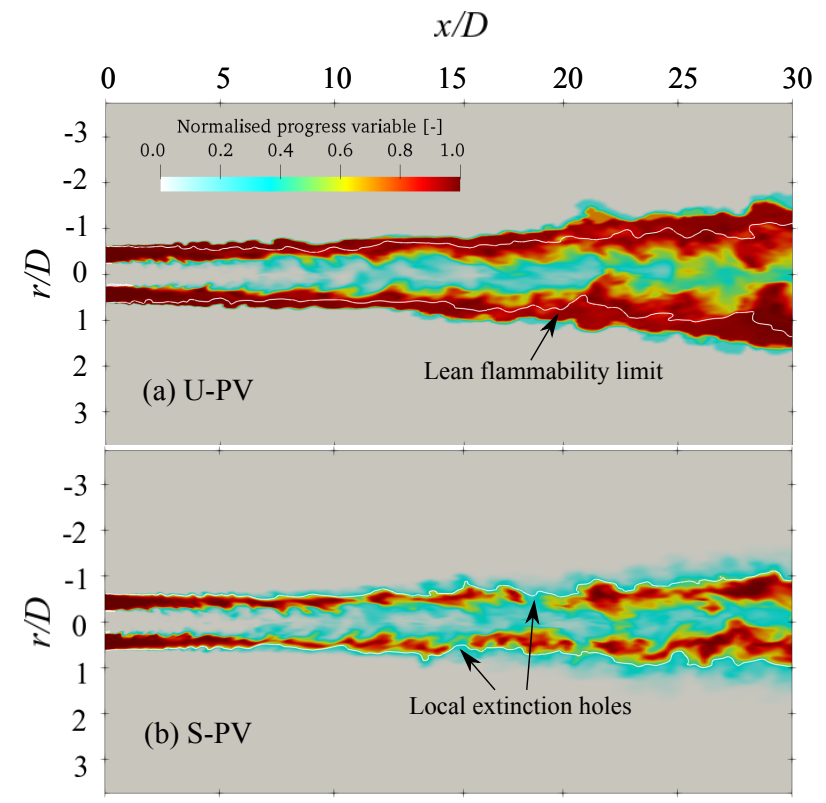

Fig. 8: Mid-plane snapshot of normalised progress variable computed using the (a) U-PV and (b) S-PV approaches for the Lr75-80 case.

This implies that the two approach may have different capabilities of capturing the local extinction behaviours, which is discussed further in later sections.

Some under-predictions of mean temperature are observed at $x / D=5$ for all three cases on the pilot side $(r>6 \mathrm{~mm})$, which may be partly caused by the instability discussed in Fig. 5. As one moves downstream, both LES approaches tend to over-predict the temperature. This is more evident at $x / D=30$ for the Lr75-103 case, which has $90 \%$ of the blow-off velocity. At the same location both LES approaches under-predict the oxygen in respect to measurements (see Supplementary Material), thus suggesting a faster burning in respect to the experiment. For the other cases the LES results match the measurements very well or show some over-predictions for $r>10 \mathrm{~mm}$ depending on the measurement set 
they are compared to. Overall, the LES results show good accuracy both in terms of mean and rms temperature, with moderate to intense local extinctions occurring in these flames. This suggests that the simple unstrained flamelet model is able to predict first and second order statistics independently of these occurrences, and this aspect will be investigated in more details in the next sections.

\subsubsection{Comparison of $\mathrm{CO}$ mass fraction}

To further assess the model abilities to capture the flame structure, CO mass fraction from LES and experiments are compared in Fig. 9. Predictions from UPV and S-PV approaches are very similar to each other at all locations for all three cases except $x / D=5$. At this location the U-PV approach prediction is slightly closer to the experimental results. In particular, a bump is observed at $r \approx 8 \mathrm{~mm}$ for the S-PV approach, which is not seen in the results for the U-PV approach. This bump is produced numerically at the pilot/coflow interface as mentioned earlier in Section 3 and suggests that a weak, spurious flame is still present for the S-PV approach. Moreover, the bump is more evident for the smaller velocity case, Lr75-57, which shows the stronger instability at the interface as seen in Fig. 5. As under-estimation of temperature was also observed at the same axial and radial locations, these differences suggest a strong influence of the pilot/coflow interface on the results. Another interesting aspect is observed for $x / D=1$. LES results at this location compare well with measurements up to $r \approx 4 \mathrm{~mm}$ (main jet/pilot interface), with the S-PV showing better estimation than the U-PV approach, but both significantly under-predict $\mathrm{CO}$ for larger $r$ values corresponding to the pilot region. As the experimental values are above equilibrium and mixture fraction at the same location was well predicted by the LES, this suggest that the 5-gases pilot mixture in the experiment is not fully burnt and further reactions are still 


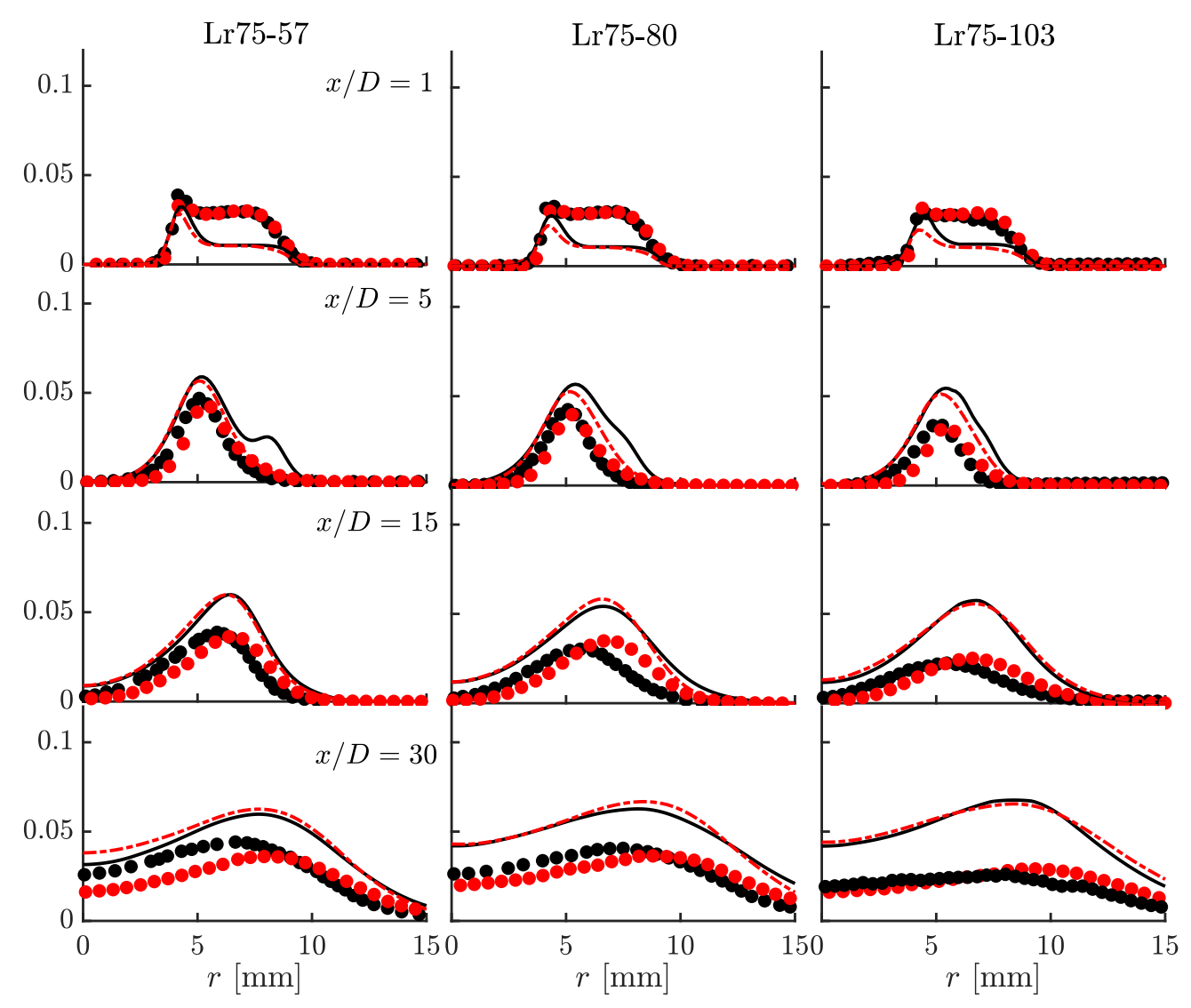

Fig. 9: Radial profiles of CO mass fraction from experiment $(\bullet$ [47], $\bullet$ [48]) are compared to LES results (S-PV, — and U-PV, - - - ) at various axial locations.

occurring downstream. Although a strong effect is not expected at the jet/pilot interface, this incomplete burning may affect the reaction-diffusion processes at the pilot/coflow interface. It is possible that this in turn affects the instability observed in Fig. 5 and thus partly explains the differences observed for temperature between LES and experimental data at the next downstream locations. Nevertheless, further investigations are needed to understand the instability at the pilot/coflow interface. One way to quantify this instability is to measure the pilot 
penetration height (where the pilot stream merges with the main jet), which can be used for LES validation. This can be explored in a future study. At further downstream locations, $x / D=15$ and $x / D=30$, the LES tends to over-estimate the $\mathrm{CO}$ in respect to measurements, and this over-estimation increases from case Lr75-57 to Lr75-103. These over-predictions are expected from a flamelet model and better predictions could be achieved by using a transport equation, which is not the focus of this study. Another source of error could be related to the tabulation method used. As has been reported by $\mathrm{Wu}$ and Ihme [56], premixed flamelets tend to give higher $\mathrm{CO}$ concentration than non-premixed flamelets even though the mixture fraction and temperature predictions are similar. This is also found in the present study as $\mathrm{CO}$ concentration is well captured in the upstream premixed burning region but significantly over-estimated in the downstream where the nonpremixed mode is dominant. Nevertheless, the present LES predicts the correct qualitative behaviour and the maximum over-prediction is less than 2.5 times the value from experiment, observed for case $\operatorname{Lr} 75-103$ at $x / D=30$.

In summary, the following points are made for the prediction of the statistical features of the flames simulated: i) overall good agreement with the measurements is obtained for both LES approaches used; ii) for the three-stream problem, the U-PV approach naturally avoids the spurious flame at the pilot/coflow interface, whereas additional treatment (tracking the fuel) is required for the S-PV; iii) the pilot penetration is over-predicted by the U-PV leading to higher mean temperature estimation in the downstream; and iv) evident local extinction spots are seen for the S-PV but not for the U-PV approach. With the current data available, however, it is difficult to assess which of the two numerical approaches, S-PV 
and U-PV, is preferable in a general sense. The latter seems to better interpret the physics at the pilot/coflow interface and deal with the three-stream problem. However, it seems quite problematic for partially premixed flames burning over the entire flammable range, because scaling the progress variable on the fly for table lookup causes significant numerical errors near the flammability limits, which has a major impact on the downstream flame local extinction behaviour. This is further demonstrated in the Supplementary Material. Since here the focus of this study is on the prediction of local extinctions, the S-PV approach is chosen for the analysis in the following sections to showcase the capabilities of flamelet models in this aspect.

\subsection{Analysis of local extinctions}

The overall good agreement observed for the comparisons with statistics in the previous section (also see Supplementary Material) suggests that the unstrained flamelet model used for this study is able, at least for what concerns main statistics, to partly take into account the effect of local extinctions. These have in fact been observed in the experiment for all three flames of Table 1, and the number of occurrences increase with the bulk velocity.

The presence of a local extinction can be qualitatively identified by appearance of 'holes' where the reaction zone suddenly becomes spatially discontinuous. The presence of such a hole can be spotted in the bottom branch of flame Lr75-103 in Fig. 3, at about $x / D=8$. A better assessment can be done by investigating $\mathrm{OH}$ mass fraction contours as these are indicative of reaction zones. $\mathrm{OH}$ experimental data is unfortunately not available for the specific flames under investigation. However, this data is available for a similar experimental configuration [46] with a larger mixing distance of $L r=100 \mathrm{~mm}$ (see Fig. 1a) and three flames (shown 

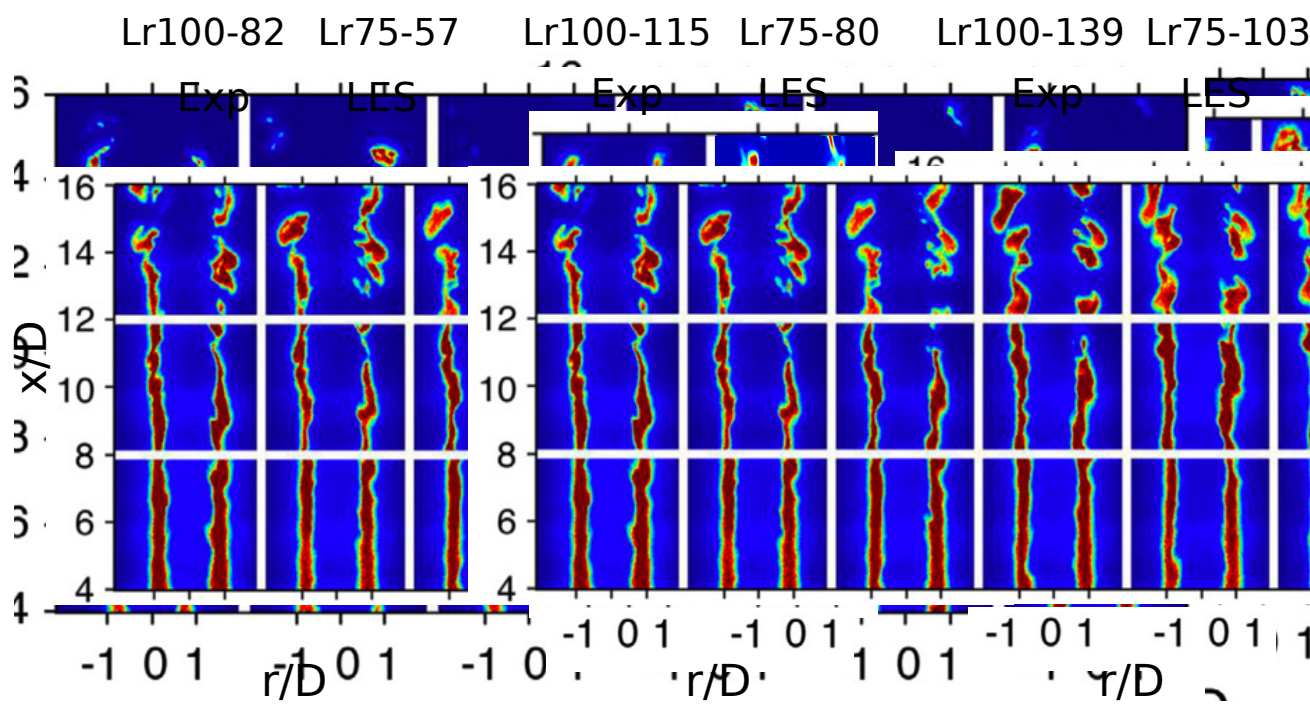

Fig. 10: Midplane contours of $\mathrm{OH}$ mass fraction from LES for the three cases of Table 1 are compared to experimental contours [47] for three flames having the same blow-off velocity ratio $U_{b} / U_{\text {b.o. }}$ and a similar configuration.

in Fig. 1b) having the same blow off velocity ratio $U_{b} / U_{\text {b.o. }}$ of those in Table 1 . Thus, a qualitative comparison between LES results and measurements is possible for the purposes of this analysis. This comparison is shown in Fig. 10. For the $50 \%$ blow-off case (left in the figure), the flame does not exhibit extinctions up to $x / D \approx 12$ and the LES results compare qualitatively well with those from experiment, although the $\mathrm{OH}$ zone in the LES looks shorter in the radial direction. Clear presence of extinctions is observed for more downstream position in the experiment. However, local extinctions are not seen for $x / D>12$ in the LES. For the $70 \%$ blow-off case (centre in the figure) evident local extinctions occur already from $x / D \approx 9$, and they are clearly observable also in the LES data. For more downstream locations $(x / D>12)$ the $\mathrm{OH}$ distribution appears to be very intermittent in both LES and experimental results as compared to the 50\% blow-off 
Lr75-57
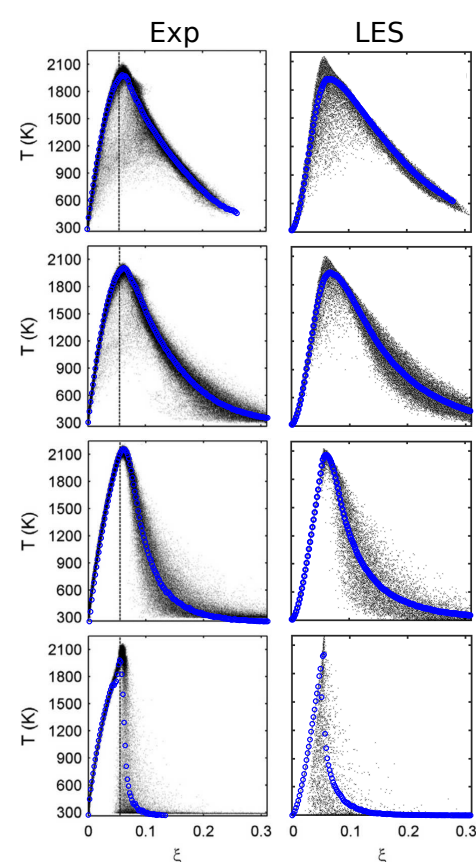
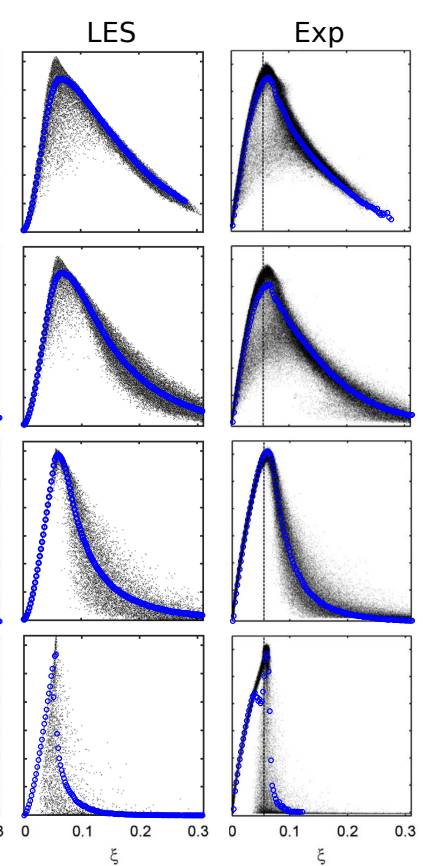

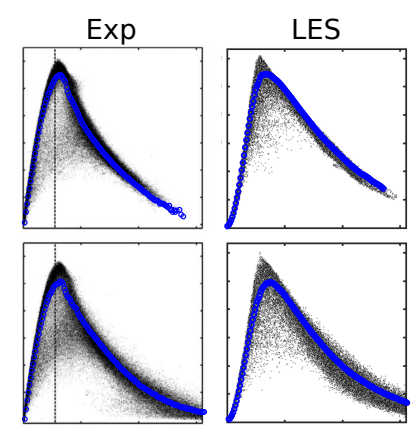

Lr75-80
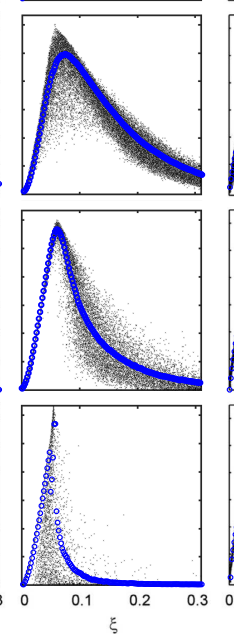
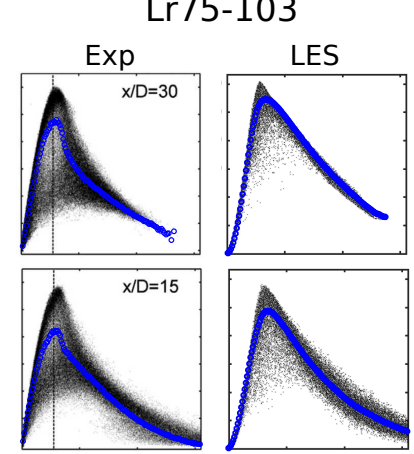

Fig. 11: Scatter plots of temperature versus mixture fraction from LES (S-PV) and experiment [47] are shown for the three flames of Table 1 at various axial locations. Circles represent the conditional mean values and vertical dashed lines indicate the stoichiometric mixture fraction.

case. For further increase of blow-ff ratio (90\%, right in the figure) local extinctions start to occur even more upstream as one would expect, $x / D<8$, and this trend is again captured by the LES. For axial locations $x / D>8$ the $\mathrm{OH}$ zone becomes very distributed in the experiment. Clear indications of local extinctions is observed also for the LES in this case; however, the flame structure appears to be more 'robust', i.e., the contour patches are less broken compared to experiment, although this could be partly attributed to particular realisations of the LES and measurement data.

The above analysis shows that the unstrained flamelet model is able to pre- 
dict, at least qualitatively, occurrences of local extinctions, and the reasons for this will be discussed in the next section. A more quantitative analysis can be performed by examining the scatter plots of temperature versus mixture fraction. These are shown and compared to experimental data in Fig. 11 for the three flames in Table 1. The first observable point in this figure is that compared with other locations, at $x / D=1$ the rich-side distribution of experimental scatters is quite different showing a vertical transition from high to low temperatures. This is seen for all three flames indicating a premixed burning mode, as opposed to the nonpremixed behaviour of gradual temperature decrease towards larger mixture fraction at the downstream locations. For the LES results, a generally larger scatter in the mixture fraction space is observed, which is partly due to the filtering nature of LES. The vertical transition at $x / D=1$ is less obvious but still observable, and the downstream switching to non-premixed mode is also captured in the LES.

Local extinctions can be identified Fig. 11 by points at relatively low temperature around the stoichiometric line, as the temperature unavoidably drops at an extinction spot. For all three flames, local extinctions become very frequent only for $x / D>5$, which is consistent with the analyses conducted in the previous sections. The LES data compare very well to experimental data for $x / D=1$ and $x / D=5$, although it exhibits more scatter on the rich side. On the lean side the scatter is very low in both LES and experiment, but the LES temperature in the $T-\xi$ plot starts with a convex angle rather than concave as for the experiment, which may be due to differential diffusion effects not taken into account in the LES. At more downstream positions presence of relatively low temperatures near the stoichiometric line (and thus increasing scatter) is indicated by the LES results for all flames, with an increasing tendency as the velocity approaches 
blow off (Lr75-57 to Lr75-103 cases), and thus the correct qualitative behaviour is captured in the LES. However, occurrences of local extinction are significantly fewer than in the experiments, which is particularly evident for the Lr75-103 case (90\% blow-off speed) as indicated by the higher conditional mean temperatures at $x / D=15$ and 30 in the LES. This is also consistent with the behaviour observed in Fig. 10 for the same flame. Similar results (not shown) were observed using a refined mesh, eliminating the potential grid dependency of local extinctions. Thus, it suggests that although the LES approach used here can reproduce the occurrence of local extinctions and the qualitative trend with increasing bulk velocity, it still has limitations when a quantitative assessment is performed. This is further discussed in the next section. The lower level of local extinctions observed here in the LES explains the over-estimated downstream temperature seen earlier in Fig. 7 for the Lr75-80 and Lr75-103 cases.

The experimental datasets $[47,48]$ also present estimation of a burning index. This was defined as

$$
\mathrm{BI}(x)=\left\langle\frac{T(x)-T_{u}}{T_{b}-T_{u}}\right\rangle_{0.058<\xi<0.068}
$$

where $T_{u}$ and $T_{b}$ are unburnt and burnt temperatures being 294 and $2100 \mathrm{~K}$ respectively, and the brackets indicate a sectional average at a fixed axial position. This quantity was averaged using only bins within $0.058<\xi<0.068$ in order to include only reacting values in the average. Only the rich side was considered to exclude the pilot gases (at stoichiometry) and low-temperature non-reacting mixture of pilot gas and coflow air from the lean side. The same burning index is computed form the LES results and compared to measurements in Fig. 12. The the low values of $\mathrm{BI}$ observed for $x / D<5$ are not due to local extinctions but the unreacted mixtures [47] in the main jet flow. The LES results compare well to 


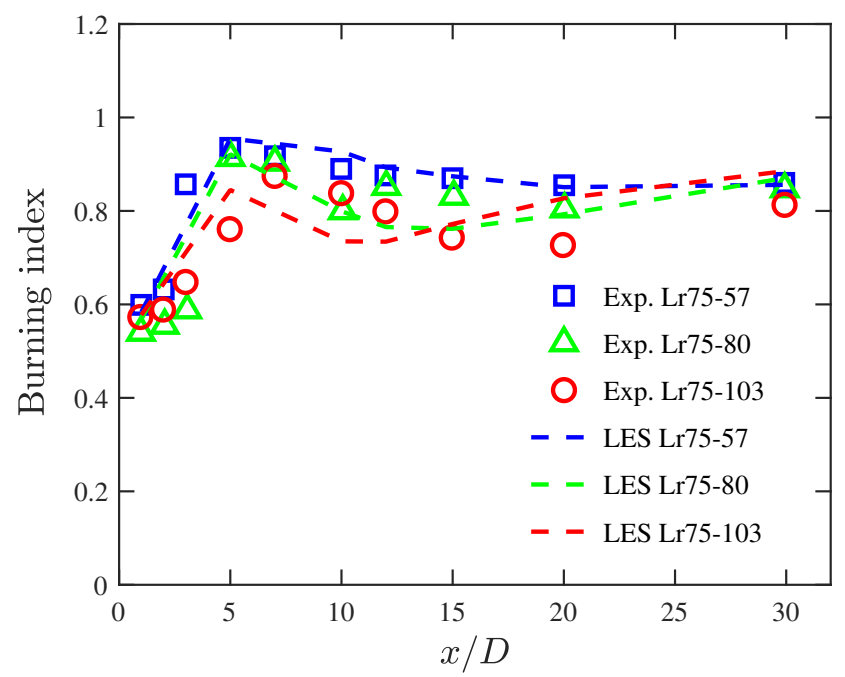

Fig. 12: Comparisons of burning index, computed using Eq. (7), form LES and experiments [48].

measurements at these locations. As one moves downstream, all three cases of Table 1 show a decrease of $\mathrm{BI}$, indicating that local extinctions occur between about 5 and $20 D$. Further downstream the flame reignites leading to an increase of BI and this is seen for all three cases. The BI decreases more for flames having higher blow-off ratio as one would expect, and measurements show that a local minimum occurs at about $x / D=20$, suggesting that most of the extinctions occur around this axial location. This decrease-then-increase trend is qualitatively captured in the LES for all three cases. However, the position of the local minimum seems to occur earlier in the LES. In particular, for case Lr75-103 having 90\% blow-off velocity, the LES significantly over-predicts the BI for $x / D>15$. This may be partly due to the fact that the LES uses a database of premixed flamelets while the balance is very much in favour of the non-premixed mode downstream as observed in the experiments [47] and discussed in section 4.1. The other reasons is 
associated with limitations of unstrained flamelets in predicting local extinctions, which is further discussed in the next section.

\section{Discussion}

The analysis carried out in section 4.2 shows that the unstrained flamelet approach used in this work is able to predict the first and second order flame statistics with good accuracy for the partially premixed jet flames with inhomogeneous inlets listed in Table 1. This is with the strong local extinctions occurring frequently for $x / D>5$ [47] in the Lr75-80 and Lr75-103 cases, implying that the LES model used here performs quite well in presence of extinctions. The largest discrepancies were observed for $x / D=5$ and $x / D=30$, see for example the temperature comparison in Fig. 7. In the first case the differences are caused by the instability at the pilot/coflow interface, as discussed in section 4.2, rather than the mis-prediction of local extinctions because local extinction is not significant at this position. In the second case, by contrast, local extinctions are evident especially for the case Lr75-103. The less severe local extinction in the LES can explain the over-prediction of temperature observed at $x / D=30$, and the corresponding under-prediction of oxygen (see Supplementary Material). However, the analysis conducted in section 4.3 shows that the simple unstrained flamelet model used here is able to capture qualitatively local extinctions, but with some quantitative limitation which is observed for case Lr75-103, the closest to blow-off condition. This suggests that the amount of local extinctions is not increasing at the correct rate as in the experiment. As a consequence, the model used is unable to predict the complete blow-off at the measured bulk velocity. This was verified by performing additional LES (not shown) with the ratios of $U_{b} / U_{\text {b.o. }}=100 \%$, 
$120 \%$ and $150 \%$. For all these cases no blow-off was observed in the LES, although the extent of local extinctions was observed to increase. Also, for higher speed cases the non-premixed mode can be more dominant at downstream positions, for which the thermochemical state may not be accurately represented by the premixed flamelets. Note that the corresponding computed mixture fraction agrees well with experimental data in Fig. 6 because mixture fraction is a passive scalar and can only be indirectly affected by the burning mode via density variations. Despite this limitation, it is surprising that a simple unstrained flamelet model can at least qualitatively predict occurrences of local extinctions before the flame quenching. Possible reasons for this are discussed next.

Both experimental works in [47] and [48] show that the scalar dissipation rate (SDR) of mixture fraction plays a fundamental role in the formation of local extinctions, independently of the progress variable SDR. Thus, this gives a chance to the model used to predict the local extinctions despite subgrid processes on the flame such as strain are not taken into account. Differently from the progress variable, the SGS processes associated with mixture fraction are relatively small and thus the LES may be capturing the correct physics at the resolved level. To demonstrate this, three-dimensional measurements of mixture fraction SDR available in the experimental dataset in [48] are compared to the computed SDR in Fig. 13. Both the resolved and the total (resolved plus SGS contribution estimated using linear relaxation model) SDR from LES are shown. Note that the diffusion constant was estimated in the experiment as (expression gives CGS units)

$$
\mathcal{D}_{\exp }=-0.12013+0.74818 \frac{T}{1000}+1.1631 \frac{T^{2}}{1000^{2}}
$$

and thus an additional curve is shown (only for the Lr75-80 case) to assess its effect as compared to the results obtained using the diffusion constant from the LES. 

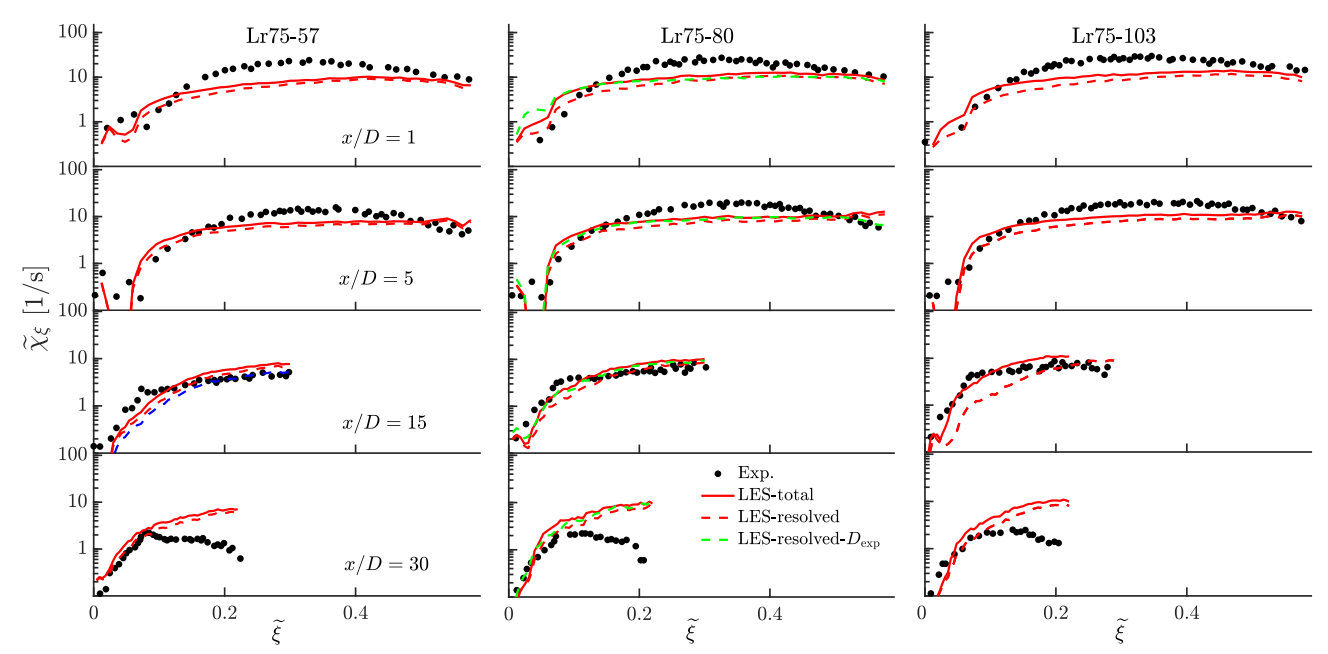

Fig. 13: Radial profiled of mixture fraction scalar dissipation rate (SDR) from experiment [48] (symbols) are compared to those from LES (lines) at different axial locations.

As can be observed in the figure, using the estimated constant leads to slightly lower SDR values as compared to the total SDR, and thus effect of diffusion coefficient is negligible. The LES predicts the correct SDR behaviour in the flammable region at all positions for all three flames. A reasonably good agreement is also seen towards the rich region in the jet core, i.e., $\xi>0.1$ except for the last downstream position at $x / D=30$ where the SDR is significantly over-predicted for all three cases. This is probably due to the generally over-estimated temperature leading to higher diffusion coefficient at this position. It is noticeable that the SGS contribution to the SDR of mixture fraction seems to play an important role at $x / D=15$ (where local extinctions occur) in particular for the higher velocity case, Lr75-103. Besides, for all three flames considerable under-prediction of SDR is seen in near field at $x / D=1$ and 5 for $\xi>0.2$. As temperature in this region is captured well (see Fig. 7), this deviation is attributed to the SGS 
model for the unresolved mixture fraction SDR. Nevertheless, first and second order statistics of mixture fraction shown in Fig. 6 show a very good agreement between LES and experiment, suggesting that these differences in the SDR do not affect the statistics. However, this also suggests that the reason behind the LES model being able to predict qualitatively the local extinctions has deeper roots. As the SGS contribution at $x / D=15$ is significantly stronger for flame Lr75-103 than for the other flames in Fig. 13, which seems to be correlated with a stronger under-prediction of temperature at the same location (see Fig. 7), it is more likely that the model ability of predicting local extinctions is attributed to the resolved scales. For instance, the resolved strain can influence the flame through filtered progress variable equation and change the burning rate. Also, the SGS SDR of mixture fraction (modelled) plays a role in the model via the subgrid variance equation. However, whether there is a mechanism within this simple unstrained model that dictates the local extinction in LES, and what are the possible effects of SGS strain and non-premixed thermochemical state, are unclear at this stage. Further analyses on the SDR are given next to shed more light on this.

Figure 14 shows the probability density function of mixture fraction SDR for various axial locations for cases Lr75-80 and Lr75-103. The PDFs are normalised by their maximum for easiness of comparison. It can be seen that the peak position is well captured for all axial locations, except for $x / D=5$ where it is overpredicted. Since at this position local extinctions are not dominant, this behaviour is probably related to the pilot effect discussed in section 4.2. At downstream positions, $x / D=15$ and $x / D=30$, the LES predicts the correct PDF shape and the peak position is also very well captured. This may explain why the main statistics are predicted well by the LES. However, the LES PDFs are significantly narrower 

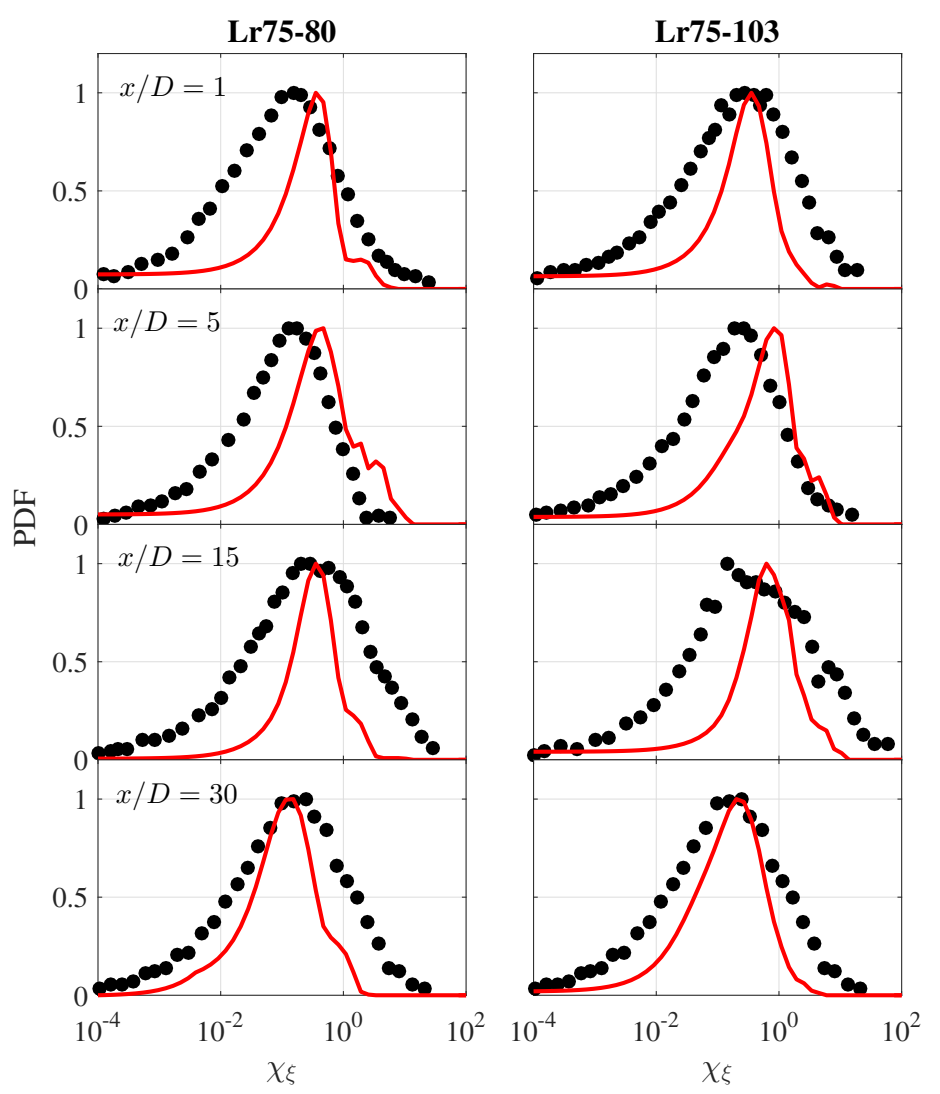

Fig. 14: PDFs (normalised by their maximum value) of total (resolved+SGS) mixture fraction scalar dissipation rate, $\chi_{\xi}$ (unit s ${ }^{-1}$ ), from experiment (symbols) are compared to PDFs obtained from LES.

than those in the experiment for both flames and at all locations. This implies that off-centred events are much less likely to happen in the LES as compared to the experiments. Higher-than-average SDR events are under-predicted in the LES as observed for $x / D=15$ and 30 in the figure, although the computed mean is generally larger than the measured one for large SDR values in Fig. 13. These comparisons indicate that the local high SDR events could not be resolved in the LES and/or that the SGS SDR model used is incapable of capturing these events. 


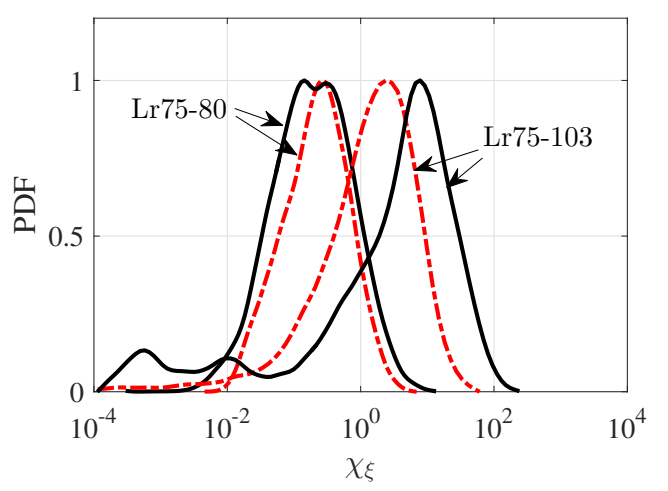

Fig. 15: PDFs (normalised by their maximum value) of total (resolved+SGS) mixture fraction scalar dissipation rate, $\chi_{\xi}$ (unit s ${ }^{-1}$ ), are shown for the regions $0<x / D<5(-)$ and $x / D>5$ $(-\cdot-)$ for cases Lr75-80 and Lr75-103 of Table 1.

Since high SDRs extinguish the flame (from the S-curve), their fewer occurrences in the LES may explain the under-predicted local extinctions at these axial positions.

As local extinctions start to occur frequently for $x / D>5$, PDFs of $\chi_{\xi}$ from LES for the regions $0<x / D<5$ and $x / D>5$ are examined separately in Fig. 15. First of all, $\chi_{\xi}$ generally increases with the bulk velocity and this is expected because of higher gradients of mixture fraction for the Lr75-103 case (less homogeneous mixing due to smaller residence time). For the Lr75-80 case with $70 \%$ of the blow-off velocity, there is no significant change in the spacial behaviour of SDR before and after $x / D=5$; however, a noticeable shift towards lower values is observed as one moves downstream for the Lr75-103 case. This is because, as the level of local extinction increases, the lower temperature field results in reduced scalar diffusivity and thus a shift in the PDF towards lower $\chi_{\xi}$ values. However, since the same behaviour is not observed for the lower blow- 
off ratio case, additional effects may be accompanying the occurrence of local extinctions when the flame approaches blow-off.

The above analysis indicates that the unstrained flamelet model may be capable of capturing local extinctions when the processes related to the mixture fraction SDR are captured, at least for what concerns the resolved scales. This further suggests that, although the linear relaxation model used for $\widetilde{\chi}_{\xi \text {,sgs }}$ yields correct statistics, improvements of this modelling could potentially lead to better predictions in presence of local extinctions. However, the analysis conducted above assumes that the mixture fraction SDR is the principal quantity responsible for local extinctions for the investigated cases, which follows from observations in the experimental work [47]. The flamelet modelling used for this work does not consider the subgrid effect of strain on the flame. Although this was shown to be small for stable flames and well-resolved LES [43, 44], it is likely to play a role in close to blow-off conditions and further analyses will be conducted on this in a future study. Moreover, it was assumed that the SDR of the progress variable does not influence significantly the local extinctions. Although strong correlation between progress variable and mixture fraction is not expected at the SGS level, the latter can be still indirectly affected by the progress variable.

\section{Summary \& Conclusions}

A LES model based on unstrained premixed flamelets and with a revised formulation for the SGS variance of progress variable has been employed to simulate three partially premixed flames having $50 \%, 70 \%$ and $80 \%$ blow-off velocity respectively. Two formulations using scaled and unscaled progress variable, referred to as S-PV and U-PV respectively, are compared with experimental mea- 
surements extensively to investigate their capabilities of capturing the multi-mode flame structure and location extinctions. A hybrid RANS/LES method is used to deal with the turbulent inlet boundary conditions and the upstream inhomogeneous mixing is captured very well for all three cases. Further analyses are conducted to assess the ability of the modelling to predict local extinctions observed in the experiments. The main findings of this work are summarised as follows.

- The LES results obtained using both the S-PV and U-PV approaches show good agreement with the measurements for the statistics of a wide range of flame/flow quantities. The flame structure is well captured for all three cases simulated in presence of low, moderate and high occurrences of local extinctions. The most significant deviations are observed for two distinct axial locations $x / D=5$ and $x / D=30$ for different reasons. Further examination of the results reveals that the deviation at $x / D=5$ is mainly attributed to a flow instability residing in the shear layer between the pilot and air coflow streams. This instability is found to be dependent on the bulk velocity of the main jet and thus exhibits different behaviours among the three flames investigated. It is well predicted for the two cases with relatively low velocities but not for the close to blowoff case where the temperature at $x / D=5$ is considerably under-estimated indicating a weaker pilot penetration compared to the experiment. Further investigation is required to shed more light on this flow instability and its effects on the flame structure and local extinctions. For the second axial location at $x / D=30$, deviations are correlated to under-predictions of oxygen and are more pronounced for the higher velocity cases, suggesting less local extinction occurrences (or stronger reignition) in the LES than in the experiment. This is also con- 
firmed by comparing the burning index between the LES and experiment.

- The U-PV approach is shown to better deal with the three-stream problem at the pilot/coflow boundary, whereas some weak spurious reaction is still found for the S-PV even though a fuel tracer is transported to mitigate this issue. On the other hand, the U-PV is less capable of reproducing the local extinctions. This is due to the intrinsic approximation associated with the way the flamelet lookup table is accessed. As the results obtained using these two approaches agree respectively with the two sets of measurements, it is not possible to conclude which one is preferable for general practice except for the specific scenarios discussed above. However, it has been clearly demonstrated in this work that the S-PV is the favourable candidate for the prediction of local extinctions.

- The unstrained flamelet approach used in this study is shown to capture local extinctions up to a certain extent. This ability seems to be associated with a satisfactory prediction of the scalar dissipation rate of the mixture fraction, which was indicated in the experiments to have a major role for the flames investigated. A comparison with the measured scalar dissipation rate shows that it is well captured for all cases except the non-reacting jet core region, where significant under-prediction is observed due to the under-resolved mixture fraction gradients. The computed PDF of scalar dissipation rate also agrees quite well with the measurements for various axial locations despite a generally narrower distribution.

Although the LES approach used in this study is able to qualitatively capture the increasing trend of local extinctions as the bulk velocity increases, it requires 
further development in order to quantitatively reproduce the same amount of local extinctions as in the experiments, and to eventually predict the blow-off at the correct bulk velocity. Nevertheless the statistics predicted by the LES are satisfactory indicating that the simple unstrained approach is useful to provide at least statistical information for flames with local extinctions. Possible improvement could be achieved by studying ad hoc closures for the SGS scalar dissipation rate of mixture fraction, or alternatively including SGS straining effects in the reaction rate closure. These will be investigated in future studies.

\section{Acknowledgement}

This work used the ARCHER UK National Supercomputing Service (http://www.archer.ac.uk) using computing time provided by EPSRC under the UKCTRF (e305). Part of this work has also been performed using resources provided by the "Cambridge Service for Data Driven Discovery" (CSD3, http://csd3.cam.ac.uk) system operated by the University of Cambridge Research Computing Service (http://www.hpc.cam.ac.uk) funded by EPSRC Tier-2 capital grant EP/P020259/1. The authors thank Dr M. Staufer and Dr S. Stow from Rolls-Royce Plc. for helpful discussions. ZXC and NS acknowledge the support of Mitsubishi Heavy Industries, Takasago, Japan. RSB acknowledges support from the U.S. Department of Energy, Office of Basic Energy Sciences, Division of Chemical Sciences, Geosciences, and Biosciences. Sandia National Laboratories is a multi-mission laboratory managed and operated by National Technology and Engineering Solutions of Sandia, LLC., a wholly owned subsidiary of Honeywell International, Inc., for the U.S. Department of Energy's National Nuclear Security Administration under contract DE-NA-0003525. 


\section{Appendix A. A hybrid RANS/LES approach for turbulent inlet boundary conditions}

For this burner configuration, the level of mixture inhomogeneity at the main jet exit dictates the flame stability and combustion regimes downstream [46, 47]. Therefore, it is of paramount importance to obtain the correct turbulent mixing profiles at the nozzle exit before any model assessment can be made. These profiles (mean and rms of $\xi$ at $x / D=1$ ) were observed to be very sensitive to the turbulence level at the domain inlets for $\mathrm{CH}_{4}$ and air, which are located $100 \mathrm{~mm}$ upstream of the jet exit (see Fig. 2). Thus, an inlet turbulence generator based on the synthetic eddy method $[80,86]$ is used to mimic the fully developed turbulent pipe flows coming into the LES domain. This inflow generator requires detailed turbulence characteristics upstream of the inlet, namely the mean profiles of velocity, Reynolds stress tensor and the streamwise integral length scale. To obtain these quantities prior to the LES, a 3D steady RANS simulation with the transported Reynolds stresses turbulence model was performed for two concentric pipes. A sufficient length of $0.5 \mathrm{~m}$ was used for the fuel and air flows to fully develop before they start mixing at the inner pipe exit. A typical inflow generation flowchart is shown in Fig. A.1 for the Lr75-80 case. As illustrated in the figure, the RANS data on the transverse plane $25 \mathrm{~mm}$ upstream of the inner pipe exit, which is the LES inlet boundary, are taken for the turbulence generator. Radial profiles of normalised mean velocity, rms and integral length scale on this plane are shown in Fig. A.2 for the Lr75-57 and Lr75-103 cases. The mean axial velocity has parabolic profiles as one would expect for fully developed pipe flows. The rms values and integral length scales also fall within the typical ranges and show the correct behaviour in their correlations. These RANS data on the trans- 


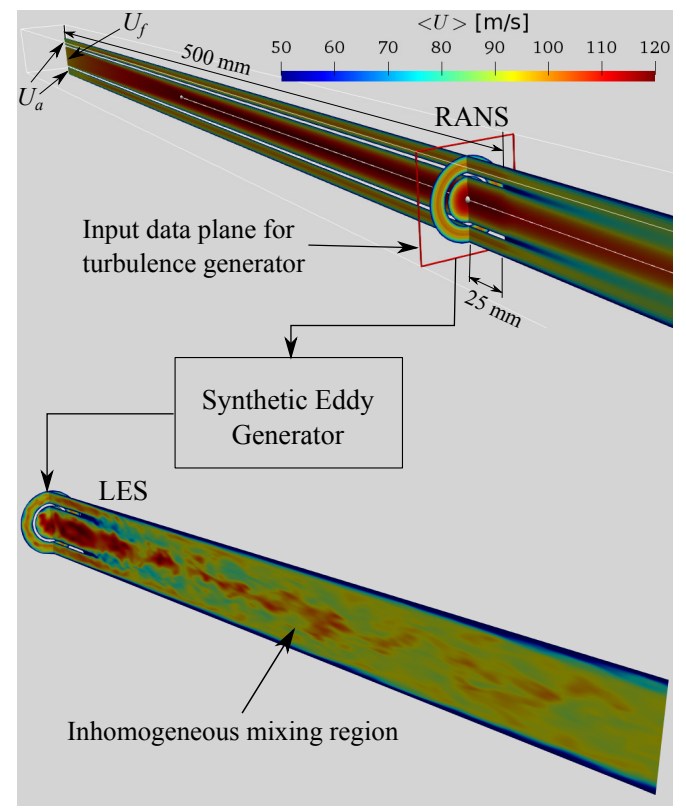

Fig. A.1: Schematic illustration of the turbulent inflow generation approach. Axial velocity contours are shown for the Lr75-80 case. $U_{f}$ and $U_{a}$ are the top-hat bulk velocities listed in Table 1 for the fuel and air inflows respectively.

verse plane (shown in Fig. A.1) are interpolated onto the LES inlet plane. The inflow generator uses these time-averaged turbulence statistics to produce corresponding synthetic Lagrangian vortices on the fly for LES. The additional cost for this is about $2 \%$ for the reacting flow LES and no tuning of parameters was needed for the all the cases tested. Excellent agreement is obtained for the mean and rms mixture fraction at $x / D=1$ as shown in Fig. 6, suggesting that the hybrid RANS/LES approach used is robust, accurate and physically consistent. It also has good potential to be used in more practical systems where long upstream pipes for air flows broadly exist. 


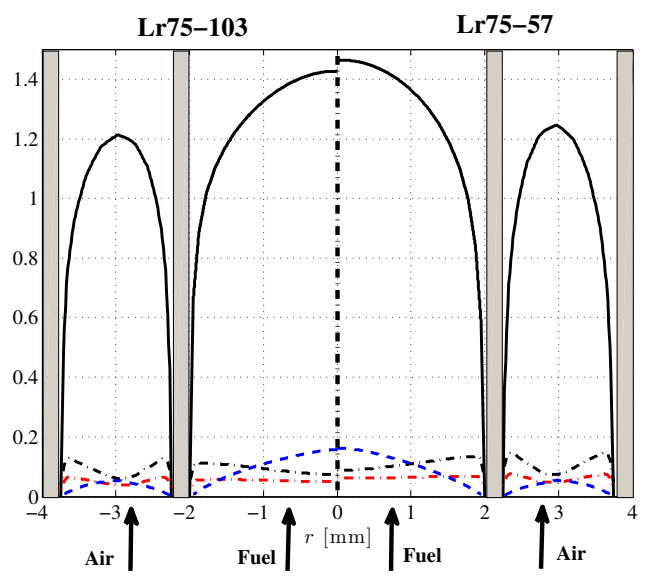

Fig. A.2: Inlet conditions used for the LES: normalised mean axial velocity $U / U_{b}(-)$, rms axial velocity $u_{\mathrm{rms}} / U_{b}(-\cdot-)$, rms radial velocity $v_{\mathrm{rms}} / U_{b}(-\cdot-)$, and normalised length scale $\Lambda / D_{e}(--)$.

\section{References}

[1] L.Y.M. Gicquel, G. Staffelbach, T. Poinsot, Large Eddy Simulations of gaseous flames in gas turbine combustion chambers, Prog. Energy Combust. Sci. 38 (2012) 782-817.

[2] N. Syred, J. M. Beér, Combustion in swirling flows: a review, Combust. Flame 23 (2) (1974) 143-201.

[3] J. E. Dec, Advanced compression-ignition engines-understanding the incylinder processes, Proc. Combust. Inst. 32 (2) (2009) 2727-2742.

[4] R.W. Bilger, Structure of diffusion flames, Combust. Sci. Technol. 13 (1976) $155-170$.

[5] F. A. Williams, Progress in knowledge of flamelet structure and extinc- 
tion, Prog. Energy Combust. Sci. 26 (2000) 657-682. doi : 10.1016/ S0360-1285(00)00012-5.

[6] M. Ihme, H. Pitsch, Prediction of extinction and reignition in nonpremixed turbulent flames using a flamelet/progress variable model: 1. a priori study and presumed pdf closure, Combust. Flame 155 (1-2) (2008) 70-89.

[7] N. R. Caetano, L. F. F. da Silva, A comparative experimental study of turbulent non premixed flames stabilized by a bluff-body burner, Exp. Therm. Fluid Sci. 63 (2015) 20-33.

[8] M. Bundy, A. Hamins, K. Y. Lee, Suppression limits of low strain rate nonpremixed methane flames, Combust. Flame 133 (3) (2003) 299 - 310.

[9] B. B. Dally, A. N. Karpetis, R. S. Barlow, Structure of turbulent nonpremixed jet flames in a diluted hot coflow, Proc. Combust. Inst. 29 (1) (2002) 1147-1154.

[10] H. Zhang, E. Mastorakos, Modelling local extinction in Sydney swirling non-premixed flames with LES/CMC, Proc. Combust. Inst. 36 (2) (2017) $1669-1676$.

[11] H. Zhang, A. Garmory, D. E. Cavaliere, E. Mastorakos, Large eddy simulation/conditional moment closure modeling of swirl-stabilized non-premixed flames with local extinction, Proc. Combust. Inst. 35 (2) (2015) 1167-1174.

[12] M. Ihme, H. Pitsch, Prediction of extinction and reignition in nonpremixed turbulent flames using a flamelet/progress variable model: 2. application in LES of Sandia flames D and E, Combust. Flame 155 (1-2) (2008) 90-107. 
[13] R.S. Barlow, J.H. Frank, Effects of turbulence on species mass fractions in methane/air jet flames, Proc. Combust. Inst. 27 (1998) 1087-1095.

[14] P. Sripagagorn, S. Mitarai, G. Kosály, H. Pitsch, Extinction and reignition in a diffusion flame: a direct numerical simulation study 518 (2004) 231-259.

[15] R. Borghi, Turbulent combustion modelling, Prog. Energy Combust. Sci. 14 (1988) 245-292.

[16] N. Peters, Turbulent Combustion, Cambridge University Press, 2000.

[17] J. P. Longwell, Flame stabilization by bluff bodies and turbulent flames in ducts, Symp. (Int.) on Combust. 4 (1) (1953) 90 - 97.

[18] E. E. Zukowski, F. E. Marble, Experiments concerning the mechanism of flame blow off from bluff bodies, Proc. Gas Dyn. Symp. Aerothermochemistry (1986) 205-210.

[19] S. Yamaguchi, N. Ohiwa, T. Hasegawa, Structure and blow-off mechanism of rod-stabilized premixed flame, Combust. Flame 62 (1) (1985) 31-41.

[20] S. Nair, T. Lieuwen, Acoustic detection of blowout in premixed flames, J. Propul. Power 21 (2005) 32-39.

[21] S. G. Tuttle, S. Chaudhuri, K. M. Kopp-Vaughan, T. R. Jensen, B. M. Cetegen, M. W. Renfro, J. M. Cohen, Lean blowoff behavior of asymmetricallyfueled bluff body-stabilized flames, Combust. Flame 160 (9) (2013) 16771692. 
[22] J. R. Dawson, R. L. Gordon, J. Kariuki, E. Mastorakos, A. R. Masri, M. Juddoo, Visualization of blow-off events in bluff-body stabilized turbulent premixed flames, Proc. Combust. Inst. 33 (2011) 1559-1566.

[23] J. Kariuki, A. Dowlut, R. Yuan, R. Balachandran, E. Mastorakos, Heat release imaging in turbulent premixed methane-air flames close to blow-off, Proc. Combust. Inst. 35 (2) (2015) 1443-1450.

[24] J. Hult, U. Meier, W. Meier, A. Harvey, C. F. Kaminski, Experimental analysis of local flame extinction in a turbulent jet diffusion flame by high repetition 2-D laser techniques and multi-scalar measurements, Proc. Combust. Inst. 30 (2005) 701-709. doi : 10 .1016/j . proci . 2004 .08 .069.

[25] B. R. Chowdhury, B. M. Cetegen, Effects of free stream flow turbulence on blowoff characteristics of bluff-body stabilized premixed flames, Combust. Flame 190 (2018) 302-316.

[26] B. Zhou, C. Brackmann, Q. Li, Z. Wang, P. Petersson, Z. Li, M. Aldén, X. S. Bai, Distributed reactions in highly turbulent premixed methane/air flames: Part I. Flame structure characterization, Combust. Flame 162 (7) (2015) $2937-2953$.

[27] H. Wang, E. R. Hawkes, B. Zhou, J. H. Chen, Z. Li, M. Aldén, A comparison between direct numerical simulation and experiment of the turbulent burning velocity-related statistics in a turbulent methane-air premixed jet flame at high Karlovitz number, Proc. Combust. Inst. 36 (2) (2017) 2045 - 2053.

[28] D. Farrace, K. Chung, S. S. Pandurangi, Y. M. Wright, K. Boulouchos, N. Swaminathan, Unstructured LES-CMC modelling of turbulent premixed 
bluff body flames close to blow-off, Proc. Combust. Inst. 36 (2) (2017) 1977 $-1985$.

[29] S. B. Pope, Turbulent Flows, Cambridge University Press, 2000.

[30] H. Pitsch, Large-Eddy Simulation of turbulent combustion, Annu. Rev. Fluid Mech. 38 (2006) 453-482.

[31] S.B. Pope, Small scales, many species and the manifold challenges of turbulent combustion, Proc. Combust. Inst. 34 (2013) 1-31.

[32] N. Peters, Laminar diffusion flamelet models in non-premixed turbulent combustion, Prog. Energy Combust. Sci. 10 (3) (1984) 319-339.

[33] D. Bradley, P.H. Gaskell, A.K.C. Lau, A mixedness-reactedness flamelet model for turbulent diffusion flames, Proc. Combust. Inst. 23 (1) (1990) 685692.

[34] J.A. van Oijen, L.P.H. de Goey, Modelling of premixed laminar flames using flamelet-generated manifolds, Combust. Sci. Technol. 161 (1) (2000) 113137.

[35] C.D. Pierce, P. Moin, Progress-variable approach for large-eddy simulation of non-premixed turbulent combustion, J. Fluid Mech. 504 (2004) 73-97.

[36] A. Donini, R.J.M. Bastiaans, J.A. van Oijen, L.P.H. de Goey, A 5-D Implementation of FGM for the Large Eddy Simulation of a Stratified Swirled Flame with Heat Loss in a Gas Turbine Combustor, Flow Turbul. Combust. 98 (3) (2017) 887-922. 
[37] P. Auzillon, O. Gicquel, N. Darabiha, D. Veynante, B. Fiorina, A filtered tabulated chemistry model for LES of stratified flames, Combust. Flame 159 (2012) 2704-2717.

[38] P. Domingo, L. Vervisch, D. Veynante, Large-eddy simulation of a lifted methane jet flame in a vitiated coflow, Combust. Flame 152 (3) (2008) 415432.

[39] Z. Chen, S. Ruan, N. Swaminathan, Large Eddy Simulation of flame edge evolution in a spark-ignited methane-air jet, Proc. Combust. Inst. 36 (2017) $1645-1652$.

[40] N. Peters, Laminar flamelet concepts in turbulent combustion, Symp. (Int.) on Combust. 21 (1986) 1231-1250.

[41] C. Meneveau, T. Poinsot, Stretching and quenching of flamelets in premixed turbulent combustion, Combust. Flame 86 (1991) 311-332.

[42] S. A. Filatyev, J. F. Driscoll, C. D. Carter, J. M. Donbar, Measured properties of turbulent premixed flames for model assessment, including burning velocities, stretch rates, and surface densities, Combust. Flame 141 (1) (2005) $1-21$.

[43] I. Langella, N. Swaminathan, Unstrained and strained flamelets for LES of premixed combustion, Combust. Theory Model. 20 (2016) 410-440.

[44] N. A. K. Doan, N. Swaminathan, N. Chakraborty, Multiscale analysis of turbulence-flame interaction in premixed flames, Proc. Combust. Inst. 36 (2016) 1929-1935. 
[45] A.R. Masri, Partial premixing and stratification in turbulent flames, Proc. Combust. Inst. 35 (2015) 1115-1136.

[46] S. Meares, A.R. Masri, A modified piloted burner for stabilizing turbulent flames of inhomogeneous mixtures, Combust. Flame 161 (2014) 484-495.

[47] R.S. Barlow, S. Meares, G. Magnotti, H. Cutcher, A.R. Masri, Local extinction and near-field structure in piloted turbulent $\mathrm{CH} 4$ /air jet flames with inhomogeneous inlets, Combust. Flame 162 (10) (2015) 3516-3540.

[48] H.C. Cutcher, R.S. Barlow, G. Magnotti, A.R. Masri, Statistics of scalar dissipation and reaction progress in turbulent flames with compositional inhomogeneities, Combust. Flame 194 (2018) 439-451.

[49] S. Meares, V. N. Prasad, G. Magnotti, R. S. Barlow, A. R. Masri, Stabilization of piloted turbulent flames with inhomogeneous inlets, Proc. Combust. Inst. 35 (2015) 1477-1484. doi : 10.1016/j .proci . 2014 .05 . 071.

[50] H. Wang, P. Zhang, A unified view of pilot stabilized turbulent jet flames for model assessment across different combustion regimes, Proc. Combust. Inst. 36 (2017) 1693-1703. doi : 10.1016/j .proci .2016.06.008.

[51] N. Kim, Y. Kim, Multi-environment probability density function approach for turbulent partially-premixed methane/air flame with inhomogeneous inlets, Combust. Flame 182 (2017) 190-205. doi:10.1016/j . combustflame.2017.04.020.

[52] B. A. Perry, M. E. Mueller, Effect of multiscalar subfilter PDF models in LES of turbulent flames with inhomogeneous inlets, Proc. Combust. Inst. 37 (2019) 2287-2295. 
[53] B. A. Perry, M. E. Mueller, A. R. Masri, A two mixture fraction flamelet model for large eddy simulation of turbulent flames with inhomogeneous inlets, Proc. Combust. Inst. 36 (2) (2017) 1767-1775.

[54] K. Kleinheinz, T. Kubis, P. Trisjono, M. Bode, H. Pitsch, Computational study of flame characteristics of a turbulent piloted jet burner with inhomogeneous inlets, Proc. Combust. Inst. 36 (2017) 1747-1757. doi : 10.1016/j.proci.2016.07.067.

[55] S. Galindo, F. Salehi, M. J. Cleary, A. R. Masri, MMC-LES simulations of turbulent piloted flames with varying levels of inlet inhomogeneity, Proc. Combust. Inst. 36 (2017) 1759-1766.

[56] H. Wu, M. Ihme, Compliance of combustion models for turbulent reacting flow simulations, Fuel 186 (2016) 853-863.

[57] M. Rieth, J.-Y. Chen, S. Menon, A. M. Kempf, A hybrid flamelet finiterate chemistry approach for efficient LES with a transported FDF, Combust. Flame 199 (2019) 183-193. doi : 10.1016/ J . COMBUSTFLAME . 2018.09. 019.

[58] I. Langella, Z.X. Chen, N. Swaminathan, S.K. Sadasivuni, Large-Eddy Simulation of reacting flows in industrial gas turbine combustor, J. Propul. Power 34 (2018) 1269-1284.

[59] Z.X. Chen, I. Langella, N. Swaminathan, M. Stöhr, W. Meier, H. Kolla, Large Eddy Simulation of a dual swirl gas turbine combustor: Flame/flow structures and stabilisation under thermoacoustically stable and unstable conditions, Combust. Flame 203 (2019) 279-300. 
[60] I. Langella, N. Swaminathan, R. W. Pitz, Application of unstrained flamelet SGS closure for multi-regime premixed combustion, Combust. Flame 173 (2016) 161-178.

[61] I. Langella, N. Swaminathan, F. A. Williams, J. Furukawa, Large-eddy simulation of premixed combustion in the corrugated-flamelet regime, Combust. Sci. Technol. 188 (9) (2016) 1565-1591.

[62] J.C. Massey, Z.X. Chen, N. Swaminathan, Lean flame root dynamics in a gas turbine model combustor, Combust. Sci. Technol. 191 (2019) 1019-1042.

[63] R.S. Barlow, G. Magnotti, H.C. Cutcher, A.R. Masri, On defining progress variable for Raman/Rayleigh experiments in partially-premixed methane flames, Combust. Flame 179 (2017) 117-129. doi : http: //dx . doi .org/ $10.1016 / \mathrm{j}$. combust flame .2017 .01 .027 .

[64] X. Chai, K. Mahesh, Dynamic k-equation model for large eddy simulation of compressible flow, J. Fluid Mech. 699 (2012) 385-413.

[65] R.W. Bilger, S.H. Stårner, R.J. Kee, On reduced mechanisms for methane-air combustion in nonpremixed flames, Combust. Flame 80 (2) (1990) 135-149.

[66] M. Ihme, L. Shunn, J. Zhang, Regularization of reaction progress variable for application to flamelet-based combustion models, J. Comput. Phys. 231 (2012) 7715-7721.

[67] L. Mongeau A. Najafi-Yazdi, B. Cuenot, Systematic definition of progress variables and intrinsically low-dimensional, flamelet generated manifolds for chemistry tabulation, Combust. Flame 159 (2012) 1197-1204. 
[68] K.N.C. Bray, P. Domingo, L. Vervisch, Role of the progress variable in models for partially premixed turbulent combustion, Combust. Flame 141 (4) (2005) 431-437.

[69] B. Fiorina, R. Baron, O. Gicquel, D. Thevenin, S. Carpentier, N. Darabiha, Modelling non-adiabatic partially premixed flames using flame-prolongation of ILDM, Combust. Theory Model. 7 (2003) 449-470.

[70] P. Domingo, L. Vervisch, K.N.C. Bray, Partially premixed flamelets in LES of nonpremixed turbulent combustion, Combust. Theory Model. 6 (4) (2002) $529-551$.

[71] S. Ruan, N. Swaminathan, O. Darbyshire, Modelling of turbulent lifted jet flames using flamelets: a priori assessment and a posteriori validation, Combust. Theory Model. 18 (2014) 295-329.

[72] M. Ihme, C.M. Cha, H. Pitsch, Prediction of local extinction and re-ignition effects in non-premixed turbulent combustion using a flamelet/progress variable approach, Proc. Combust. Inst. 30 (2005) 793-800.

[73] Z. Chen, S. Ruan, N. Swaminathan, Simulation of turbulent lifted methane jet flames: effects of air-dilution and transient flame propagation, Combust. Flame 162 (2015) 703-716.

[74] S. Ruan, N. Swaminathan, O.R. Darbyshire, Modelling of turbulent lifted jet flames using flamelets: a priori assessment and a posteriori validation, Combust. Theory Model. 18 (2) (2014) 295-329. 
[75] A.W. Vreman, B.A. Albrecht, J.A. van Oijen, L.P.H. de Goey, R.J.M. Bastiaans, Premixed and nonpremixed generated manifolds in Large Eddy Simulation of Sandia flame D and F, Combust. Flame 153 (2008) 394-416.

[76] N. Swaminathan, K. N. C. Bray, Effect of dilatiation on scalar dissipation in turbulent premixed flames, Combust. Flame 143 (2005) 549-565.

[77] T.D. Dunstan, Y. Minamoto, N. Chakraborty, N. Swaminathan, Scalar dissipation rate modelling for large eddy simulation of turbulent premixed flames, Proc. Combust. Inst. 34 (2013) 1193-1201.

[78] I. Langella, N. Swaminathan, Y. Gao, N. Chakraborty, Assessment of dynamic closure for premixed combustion large eddy simulation, Combust. Theory Model. 19 (5) (2015) 628-656.

[79] H. G. Weller, G. Tabor, H. Jasak, C. Fureby, A tensorial approach to computational continuum mechanics using object-oriented techniques, Computers Phys. 12 (6) (1998) 620-631.

[80] N. Kornev, E. Hassel, Method of random spots for generation of synthetic in- homogeneous turbulent fields with prescribed autocorrelation functions, Comm. Numer. Meth. Eng. 23 (2007) 35-43.

[81] U. Piomelli, E. Balaras, Wall-layer models for large-eddy simulations, Annu. Rev. Fluid Mech. 34 (1) (2002) 349-374.

[82] R. J. Kee, J. F. Grcar, M. D. Smooke, J. A. Miller, A fortran program for modeling steady laminar one-dimensional premixed flames, Report No. SAND85-8240, Sandia National Labortories, CA, USA, 1985. 
[83] W. Polifke, P. Flohr, M. Brandt, Modeling of inhomogeneously premixed combustion with an extended TFC model, J. Eng. Gas Turbines Power 124 (1) (2000) 58-65.

[84] H. Yamashita, M. Shimada, T. Takeno, A numerical study on flame stability at the transition point of jet diffusion flames, Proc. Combust. Inst. 26 (1) (1996) 27-34. doi:http://dx.doi.org/10.1016/S0082-0784(96) 80196-2.

[85] L. Tian, R. P. Lindstedt, Evaluation of reaction progress variable - mixture fraction statistics in partially premixed flames, Proc. Combust. Inst. 37 (2019) 2241-2248.

[86] N. Jarrin, S. Benhamadouche, D. Laurence, R. Prosser, A synthetic-eddy method for generating inflow conditions for large eddy simulations, J. Heat Fluid Flow 27 (2006) 585-593. 